J Org Chem. 2016 September 02; 81(17): 8050-8060. doi:10.1021/acs.joc.6b01446.

\title{
Synthesis of 1,3-Substituted Cyclobutanes by Allenoate-Alkene [2 + 2] Cycloaddition
}

\author{
Michael L. Conner and M. Kevin Brown ${ }^{*}$ \\ Indiana University, Department of Chemistry, 800 East Kirkwood Ave, Bloomington, Indiana \\ 47405, United States
}

\begin{abstract}
A method for the [2+2] cycloaddition of terminal alkenes with allenoates is presented. This process allows for the rapid synthesis of 1,3-substituted cyclobutanes in high yield under simple and robust reaction conditions.
\end{abstract}

\section{Graphical Abstract}

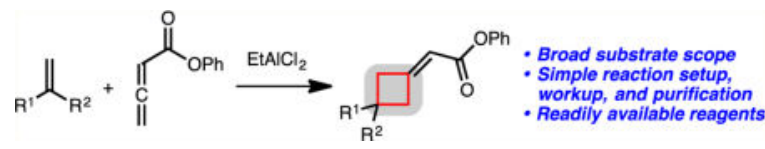

Cyclobutanes constitute an important class of molecules as they are useful intermediates in chemical synthesis and are present in a range of natural products and other biologically significant molecules. ${ }^{1-3} 1,3$-Substituted cyclobutanes are particularly important as they are commonly evaluated in drug discovery efforts (Figure 1). This may be due to several attributes of the cyclobutane ring system: (1) The spatial arrangement of the substituents is relatively well-defined due to the limited flexibility of the cyclobutane ring, which can be favorable in drug design. ${ }^{4}$ (2) In most cases, the 1,3-substituted cyclobutane core is achiral and therefore enantioselective synthesis is not required. ${ }^{5,6}$ (3) 1,3-Substituted cyclobutanes have been shown to be isosteric with aromatic ring systems in molecules of pharmacological interest. $^{7-9}$

Several methods have been reported to access 1,3-substituted cyclobutanes. Commonly used methods include (1) functionalization of commercially available 1,3-substituted cyclobutanes, (2) intramolecular alkylation/decarboxylation strategies utilizing malonate derivatives, ${ }^{10}$ and $(3)[2+2]$ cycloadditions of two $\pi$-systems. With respect to the latter, perhaps the most common method is the [2+2] cycloaddition of dichloroketene and alkenes. ${ }^{11-13}$ This process is notable in that a large number of alkenes are compatible in the reaction; however, to access 1,3-substituted cyclobutanes, an additional dehalogenation step

\footnotetext{
*Corresponding Author brownmkb@indiana.edu. ASSOCIATED CONTENT

Supporting Information

The Supporting Information is available free of charge on the ACS Publications website at DOI: 10.1021/acs.joc.6b01446.

Copies of all ${ }^{1} \mathrm{H}$ NMR, ${ }^{13} \mathrm{C}$ NMR, and appropriate 2D NMR (PDF)

The authors declare no competing financial interest.
} 
is required. Other more recent but less well-established methods include cycloadditions of thioketenes ${ }^{14}$ and an Fecatalyzed cycloaddition of dienes with terminal alkenes. ${ }^{15}$ Despite these advances, additional methods are needed, especially protocols that are simple, employ readily available reagents, and enjoy wide substrate scope.

On the basis of previous work from our lab regarding catalytic enantioselective [2 +2$]$ cycloadditions of allenoates and alkenes (Scheme 1C), ${ }^{16}$ we became interested in extending the applicability of this strategy to access a broad range of 1,3-substituted cyclobutanes by utilizing readily available achiral Lewis acid promoters. ${ }^{17}$ Prior work from Snider and Hoffman demonstrated the general reactivity of allenoates in [2+2] cycloadditions with alkenes; however, formation of 1,3-substituted cyclobutanes was limited in scope and in some cases required long reaction times (Scheme 1A and B). ${ }^{18,19}$ In our previous report, we showed that 1,3-substituted cyclobutanes could be prepared; however, the scope was again limited, and in some cases, use of trifluoroethyl 2,3-butadienoate, which can be difficult to prepare due to its volatility, was required (Scheme 1C). Over the course of our investigation, we discovered that phenyl 2,3-butadienoate (1) could be easily prepared and readily underwent Lewis acid promoted [2 +2$]$ cycloaddition with terminal alkenes using a simple Lewis acid promoter (Scheme 1D). Herein, we report the scope of this method as well as several functionalization reactions.

Early efforts led to a set of optimized conditions, which are depicted in entry 1 of Table 1. Under these conditions, the reaction is promoted by stoichiometric quantities of $\mathrm{EtAlCl}_{2}$, run in $\mathrm{CH}_{2} \mathrm{Cl}_{2}$ at room temperature (rt), and led to generation of the product in $90 \%$ yield. Illustrated in Table 1, entries 2-20 are the effects of changing reaction conditions and the nature of the allenoate on the outcome of the reaction. Several points are noteworthy: (1) Comparison of the ester group substitution clearly demonstrates the unique reactivity of phenyl 2,3-butadienoate (1) (Table 1, compare entries 1-5). The improved reactivity of phenyl 2,3-butadienoate (1) relative to that of alkyl 2,3-butadienoates is likely due to the increased electron-withdrawing nature of the phenyl ester as compared to that of alkyl esters. (2) Although the use of 1.5 equiv of $\mathrm{EtAlCl}_{2}$ led to optimal results, decreased Lewis acid loadings could be utilized (Table 1, compare entries 1 and 6-7). It should be noted that $\mathrm{EtAlCl}_{2}$ is inexpensive and easy to use. (3) The use of other common Lewis acids were not effective at promoting the cycloaddition (Table 1, entries 8-12). (4) Although $\mathrm{CH}_{2} \mathrm{Cl}_{2}$ proved to be the optimal solvent for conducting the reaction, hexanes was also effective provided that the reaction time was extended. (Table 1, compare entries 1 and 17). (5) The reaction can be carried out in concentrations higher than $0.2 \mathrm{M}$ with a modest decrease in yield (Table 1, entry 18). Additionally, the reaction could be performed utilizing only the residual solvent from the $\mathrm{EtAlCl}_{2}$ (1 $\mathrm{M}$ in hexanes) used to promote the reaction (Table 1, entry 19). (6) Finally, the reaction could easily be carried out with benchtop solvents and reagents in air with only a modest decrease in yield, which is likely due to the sensitivity of the Lewis acid to residual water in the reaction (Table 1, entry 20).

The phenyl ester allenoate was easily prepared on a large scale ( $>5 \mathrm{~g})$ utilizing straightforward Wittig olefination of in situ-generated ketene (Scheme 2). ${ }^{20}$ The allenoate can be easily handled as a liquid at room temperature; however, long-term storage must be conducted at reduced temperature $\left(-20^{\circ} \mathrm{C}\right)$ where it can be stored under $\mathrm{N}_{2}$ for greater than 
18 months with no noticeable decrease in purity. For ease of storage, we sought to identify an allenoate that could be handled and stored on the benchtop for long periods of time without the need for specialized storage requirements. After evaluating a range of allenoates, 2-naphthyl 2,3-butadienoate (2) was identified as the ideal candidate as it is a free-flowing white solid at room temperature and can be stored at ambient temperature for months. Cycloadditions with 2 provided the product with slightly reduced yield when compared to that of phenyl 2,3-butadienoate (Scheme 2B). a-Bromoallenoate $\mathbf{4}$ was also investigated in the reaction and provided yields comparable to those of the parent phenylallenoate (Scheme 2C); however, because of the ease of use and preparation, we decided to evaluate the reaction utilizing phenyl 2,3-butadienoate (1).

With these reaction considerations in hand, we turned our attention to evaluating the substrate scope of the reaction utilizing phenyl 2,3-butadienoate (1) (Scheme 3). A wide variety of terminal alkenes (both mono- and 1,1-disubstituted) undergo [2 + 2] cycloaddition in good to high yields. ${ }^{21}$ Several points regarding the substrate scope are noteworthy: (1) Unactivated terminal alkenes function well in this process. In general, yields decrease when the alkene is deactivated with an electron-withdrawing group (compare products $\mathbf{1 2}$ and $\mathbf{1 3}$ ). (2) A variety of styrene derivatives (both electron-rich and -poor) can be used to provide cycloadducts in good to high yields. (3) The reaction can be run on gram scale in high yield (see product 14). (4) The use of cyclic 1,1-disubstituted alkenes provides access to polycyclic compounds including spiro[3.3] heptane 32. (5) A limitation of this method is the lack of tolerance for substrates incorporating Lewis basic heteroatoms. For example, alkenes bearing an unprotected alcohol, or any form of nitrogen, did not function well, presumably due to Lewis acid inhibition. For this limitation to be addressed, it was demonstrated that cycloaddition could occur with functionality that would allow for the introduction of Lewis basic groups. For example, cycloadditions with Bpin- or Br-containing substrates can easily be transformed to alcohol- or amine-containing products. ${ }^{22}$ In addition, metalcatalyzed cross-coupling protocols would allow for introduction of a variety of groups.

To further demonstrate the practicality of this method, several functionalization reactions were performed (Scheme 4). Homogenous hydrogenation utilizing in situ-generated IPrCu$\mathrm{H}$ followed by reduction of the resulting ester could be performed to provide alcohol $\mathbf{3 3}$ in $73 \%$ yield and 2:1 dr (major diastereomer not determined). ${ }^{23}$ Heterogeneous hydrogenation of 14 catalyzed by $\mathrm{Pd} / \mathrm{C}$ provided the same major diastereomer of product in $86 \%$ yield with only a slight increase in dr. Reduction with $\mathrm{LiAlH}_{4}$ was only carried out to aid in the purification of the desired product from several byproducts.

Rhodium-catalyzed conjugate addition of phenyl boronic acid proceeded in good yield and 3.2:1 dr favoring syn diastereomer 34 (Scheme 4) ${ }^{24}$ Likewise, conjugate addition of $\mathrm{PhSH}$ yielded ester $\mathbf{3 5}$ with no evidence of transesterification of the phenyl ester. ${ }^{25}$ The major diastereomer observed for both conjugate addition products resulted from addition of the nucleophile from the same face of the $\mathrm{Ph}$ group. To account for this mode of addition, we propose that the puckering of the cyclobutane ring and positioning of the $\mathrm{Ph}$ group in a pseudoequatorial orientation allows for the approach of reagents from the more accessible convex face as illustrated in Scheme 4.

J Org Chem. Author manuscript; available in PMC 2017 September 02. 
In conclusion, we have developed a straightforward approach to access a wide range of 1,3substituted cyclobutanes. This method is notable in that these compounds can be accessed in a matter of hours, on a gram scale, and without the need for a specialized reaction setup. The products prepared are amenable to further derivatization due to the number of functional handles that can be incorporated.

\section{EXPERIMENTAL SECTION}

\section{General Information}

${ }^{1} \mathrm{H}$ NMR $(400,500$, or $600 \mathrm{MHz}),{ }^{13} \mathrm{C}$ NMR $(100,125$, or $150 \mathrm{MHz})$, and ${ }^{19} \mathrm{~F}$ NMR (375

$\mathrm{MHz}$ ) spectra were recorded at room temperature unless otherwise stated. Chemical shifts are reported in ppm from tetramethylsilane with the solvent resonance as the internal standard $\left({ }^{1} \mathrm{H} \mathrm{NMR} \mathrm{CDCl}_{3}: \delta 7.26 \mathrm{ppm} ;{ }^{13} \mathrm{C} \mathrm{NMR} \mathrm{CDCl}_{3}: \delta 77.2 \mathrm{ppm}\right)$. Data are reported as follows: chemical shift, multiplicity ( $\mathrm{s}=$ coupling constants $(\mathrm{Hz})$ ), and integration. Infrared spectra (IR) were obtained on an FT-IR instrument at room temperature on a $\mathrm{NaCl}$ salt plate and recorded in wavenumbers $\left(\mathrm{cm}^{-1}\right)$. Bands are characterized as broad (br), strong (s), medium (m), and weak $(\mathrm{w})$. Melting points were obtained in a capillary melting point apparatus without correction. High-resolution mass spectra were recorded on a tandem mass spectrometer with a double focusing magnetic sector-electric sector 3D ion trap.

Unless otherwise noted, all reactions have been carried out with distilled and degassed solvents under an atmosphere of dry $\mathrm{N}_{2}$ in oven- $\left(135^{\circ} \mathrm{C}\right)$ and flame-dried glassware with standard vacuum-line techniques. Reported yields are the average of at least two successful reactions unless stated otherwise. Dichloromethane, diethyl ether, and tetrahydrofuran were purified under a positive pressure of dry argon by passage through two columns of activated alumina. Toluene was purified under a positive pressure of dry argon by passage through columns of activated alumina and Q5 (Grubbs apparatus). All workup and purification procedures were carried out with reagent grade solvents in air. Standard column chromatography techniques using ZEOprep 60/40-63 $\mu \mathrm{m}$ silica gel were used for purification.

\section{Synthesis of Allenes}

Phenyl Buta-2,3-dienoate [1] -The following procedure is slightly modified from a reported literature procedure to include more detail. ${ }^{20}$ (Phenyloxymethylene)triphenylphosphorane ( $10.0 \mathrm{~g}, 25.2 \mathrm{mmol}, 1.00$ equiv) was added to a flame-dried $250 \mathrm{~mL}$ flask equipped with a stir bar and dissolved in $\mathrm{CH}_{2} \mathrm{Cl}_{2}(100 \mathrm{~mL}, 0.40 \mathrm{M}) . \mathrm{Et}_{3} \mathrm{~N}(3.50 \mathrm{~mL}$, $25.2 \mathrm{mmol}, 1.00$ equiv) was added in one portion and allowed to stir for $5 \mathrm{~min}$ at room temperature. Freshly distilled acetyl chloride $(1.80 \mathrm{~mL}, 25.2 \mathrm{mmol}, 1.00$ equiv) was added over 20 min by syringe pump as a solution in $\mathrm{CH}_{2} \mathrm{Cl}_{2}(50.0 \mathrm{~mL}, 0.50 \mathrm{M})$. Upon complete addition, the reaction was allowed to stir for $4 \mathrm{~h}$ at room temperature (note that extending the reaction time to longer than $4 \mathrm{~h}$ results in a large amount of byproduct formation as a result of chloride addition to the allenoate). The reaction was concentrated, and the resulting slurry was suspended in $150 \mathrm{~mL}$ of 1:1 $\mathrm{Et}_{2} \mathrm{O}$ :pentane, shaken vigorously, filtered through Celite, and concentrated. The crude yellow-orange residue was purified by silica gel column chromatography (2-5\% EtOAc:hexanes, gradient) to yield $3.40 \mathrm{~g} \mathrm{(21.2} \mathrm{mmol,} \mathrm{84 \%} \mathrm{yield)} \mathrm{of}$ 
the title compound as a clear colorless liquid. ${ }^{1} \mathrm{H}$ NMR $\left(500 \mathrm{MHz}, \mathrm{CDCl}_{3}\right) \delta 7.41-7.35(\mathrm{~m}$, 2H), 7.26-7.21 (m, 3H), 7.15-7.11 (m, 1H), $5.83(\mathrm{t}, J=6.5 \mathrm{~Hz}, 1 \mathrm{H}), 5.34$ (d, $J=6.5 \mathrm{~Hz}$, 2H). ${ }^{13} \mathrm{C} \mathrm{NMR}\left(125 \mathrm{MHz}, \mathrm{CDCl}_{3}\right) \delta 216.8,164.3,150.9,129.5,126.0,121.7,87.9,79.9$.

Naphthalen-2-yl 2-Bromoacetate [46]-2-Naphthol (7.21 g, 50.0 mmol, 1.00 equiv) was added to a $100 \mathrm{~mL}$ round-bottom flask, evacuated, and backfilled with nitrogen three times. $\mathrm{CH}_{2} \mathrm{Cl}_{2}(65 \mathrm{~mL}, 0.78 \mathrm{M})$ was added to dissolve the solid, and the reaction flask was cooled to $0{ }^{\circ} \mathrm{C}$. Pyridine ( $4.00 \mathrm{~mL}, 50.0 \mathrm{mmol}, 1.00$ equiv) was added, and the resulting mixture was stirred for $5 \mathrm{~min}$ to equilibrate the temperature. Bromoacetyl bromide (4.35 $\mathrm{mL}, 50 \mathrm{mmol}, 1.00$ equiv) was added slowly at $0{ }^{\circ} \mathrm{C}$ and stirred at that temperature for 15 min before removing the flask from the cooling bath and allowed the mixture to warm to room temperature for an additional $30 \mathrm{~min}$. The reaction was diluted with $\mathrm{H}_{2} \mathrm{O}(50 \mathrm{~mL})$, and the layers separated. The organic phase was washed with brine, dried over $\mathrm{MgSO}_{4}$, filtered, and concentrated under reduced pressure to yield a light brown solid (12.4 g, crude) that was used without further purification.

Naphthalen-2-yl 2-(Triphenyl- $\lambda_{5}$-phosphanylidene)acetate [47]-Prepared in the same manner as (phenyloxymethylene)- triphenylphosphorane outlined above using $\mathbf{4 6}$. Product was carried forward, and a crude off-white solid was generated.

Naphthalen-2-yl Buta-2,3-dienoate [2]—Compound 47 (5.00 g, 11.2 mmol, 1 equiv) was added to a flame-dried $150 \mathrm{~mL}$ flask equipped with a stir bar and dissolved in $\mathrm{CH}_{2} \mathrm{Cl}_{2}$ (45.0 mL, 0.25 M). $\mathrm{Et}_{3} \mathrm{~N}(1.56 \mathrm{~mL}, 11.2 \mathrm{mmol}, 1.00$ equiv) was added in one portion and allowed to stir for $5 \mathrm{~min}$ at room temperature. Freshly distilled acetyl chloride $(800 \mu \mathrm{L}, 11.2$ mmol, 1.00 equiv) was added over $20 \mathrm{~min}$ by syringe pump as a solution in $\mathrm{CH}_{2} \mathrm{Cl}_{2}(22.0$ $\mathrm{mL}, 0.50 \mathrm{M})$. Upon complete addition, the reaction was allowed to stir for $16 \mathrm{~h}$ at room temperature. The reaction was concentrated, and the resulting slurry was suspended in 100 $\mathrm{mL}$ of 1:1 $\mathrm{Et}_{2} \mathrm{O}$ :pentane, shaken vigorously, filtered through Celite, and concentrated. The crude yellow-orange residue was purified by silica gel column chromatography (2-5\% EtOAc:hexanes, gradient) to yield $1.22 \mathrm{~g}(5.80 \mathrm{mmol}, 52.0 \%$ yield $)$ of the title compound as a bench-stable white powder. IR (film): 3062 (w), 2990 (w), 1968 (m), 1735 (s), 1630 (w), 1600 (w), 1510 (w), 1465 (w), 1342 (m), 1229 (s), 1208 (s), 1133 (s), $1156(\mathrm{~s}) \mathrm{cm}^{-1} .{ }^{1} \mathrm{H}$ NMR (400 MHz, $\left.\mathrm{CDCl}_{3}\right) \delta$ 7.90-7.75 (m, 3H), 7.63-7.58 (m, 1H), 7.52-7.43 (m, 2H), $7.32-7.22(\mathrm{~m}, 1 \mathrm{H}), 5.88(\mathrm{t}, J=6.5 \mathrm{~Hz}, 1 \mathrm{H}), 5.36(\mathrm{~d}, J=6.5 \mathrm{~Hz}, 2 \mathrm{H}) .{ }^{13} \mathrm{C}$ NMR $(100 \mathrm{MHz}$, $\left.\mathrm{CDCl}_{3}\right) \delta 216.8,164.5,148.5,133.9,131.6,129.5,127.9,127.8,126.7,125.9,121.2,118.7$, 87.9, 80.0. HRMS (EI-MS): calcd for $\mathrm{C}_{14} \mathrm{H}_{11} \mathrm{O}_{2}[\mathrm{M}+\mathrm{H}]^{+}$211.0754; found 211.0751. Mp $55-57^{\circ} \mathrm{C}$.

Phenyl 2-Bromo-2-(triphenyl- $\lambda_{5}$-phosphanylidene)acetate [48]-The procedure was adapted from a known literature procedure. ${ }^{26}$

(Phenyloxymethylene)triphenylphosphorane ( $3.00 \mathrm{~g}, 7.57 \mathrm{mmol}, 1.00$ equiv) was added to a flame-dried $50 \mathrm{~mL}$ round-bottom flask, evacuated, and backfilled with nitrogen three times. $\mathrm{CH}_{2} \mathrm{Cl}_{2}(15.0 \mathrm{~mL}, 0.50 \mathrm{M})$ was added to dissolve the solid, and the reaction flask was cooled to $0{ }^{\circ} \mathrm{C}$. Bromine ( $407 \mu \mathrm{L}, 7.95 \mathrm{mmol}, 1.05$ equiv) was added as a solution in $\mathrm{CH}_{2} \mathrm{Cl}_{2}$ $(2.20 \mathrm{~mL}, 3.5 \mathrm{M})$. After complete addition, the reaction was stirred for $5 \mathrm{~h}$ at room 
temperature. The reaction was quenched with $\mathrm{H}_{2} \mathrm{O}(10 \mathrm{~mL})$ and extracted with $\mathrm{CH}_{2} \mathrm{Cl}_{2}(3 \times$ $10 \mathrm{~mL}$ ). The combined organic extracts were washed with brine, dried over $\mathrm{MgSO}_{4}$, filtered, and concentrated under reduced pressure to yield a brown solid, which was used without further purification.

Phenyl 2-Bromobuta-2,3-dienoate [4]-The procedure was adapted from a known literature procedure. ${ }^{27}$ Compound 48 ( $3.15 \mathrm{~g}, 6.63 \mathrm{mmol}, 1.00$ equiv) was added to a flamedried $50 \mathrm{~mL}$ round-bottom flask and purged three times with $\mathrm{N}_{2} . \mathrm{CH}_{2} \mathrm{Cl}_{2}(15.0 \mathrm{~mL}, 0.50 \mathrm{M})$ was added to dissolve the solid followed by $\mathrm{Et}_{3} \mathrm{~N}(920 \mu \mathrm{L}, 6.63 \mathrm{mmol}, 1.00$ equiv), and the reaction flask was cooled to $0{ }^{\circ} \mathrm{C}$. Acetyl chloride $(470 \mu \mathrm{L}, 6.63 \mathrm{mmol}, 1.00$ equiv) was added dropwise at $0{ }^{\circ} \mathrm{C}$, and the reaction was stirred at that temperature for $30 \mathrm{~min}$. The flask was removed from the cooling bath and concentrated under reduced pressure. The resulting residue was suspended in $\mathrm{Et}_{2} \mathrm{O}$ :hexanes $(1: 1,50 \mathrm{~mL})$, shaken vigorously, filtered through a pad of Celite to remove $\mathrm{Ph}_{3} \mathrm{PO}$, and concentrated. Purification of the resulting yellow liquid by silica gel column chromatography (2-4\% EtOAc:hexanes, gradient) provided $589 \mathrm{mg}$ ( $2.45 \mathrm{mmol}, 37 \%$ yield $)$ of the title compound as an off-white low melting solid. IR (film): 3004 (m), 1745 (s), 1490 (m), 1275 (s), 1260 (s), 1190 (m) cm ${ }^{-1} .{ }^{1} \mathrm{H}$ NMR $\left(500 \mathrm{MHz}, \mathrm{CDCl}_{3}\right) \delta$ 7.42-7.36 (m, 2H), 7.28-7.23 (m, 1H), 7.17-7.12 (m, 2H), $5.41(\mathrm{~s}$, $2 \mathrm{H}) .{ }^{13} \mathrm{C}$ NMR $\left(125 \mathrm{MHz}, \mathrm{CDCl}_{3}\right) \delta 212.9,160.7,151.0,129.7,126.4,121.4,84.8,83.8$. HRMS (CI-MS): calcd for $\mathrm{C}_{10} \mathrm{H}_{7} \mathrm{O}_{2} \mathrm{Br}_{1}[\mathrm{M}]^{+}$237.9624; found 237.9615 .

\section{Synthesis of Cycloadducts}

The cycloadducts were prepared utilizing the following general procedures. All reactions were run on a $0.20 \mathrm{mmol}$ scale unless stated otherwise. Purification for each compound is outlined individually.

General Procedure A-To a flame-dried flask were added 2,3-butadienoate (1.00 equiv), $\mathrm{CH}_{2} \mathrm{Cl}_{2}(0.50 \mathrm{M})$, and alkene (2.00 equiv). $\mathrm{EtAlCl}_{2}$ (1.5 equiv, $1 \mathrm{M}$ in hexanes) was added down the walls of the flask. The reaction mixture was allowed to stir at room temperature for $6 \mathrm{~h}$ before quenching with $\mathrm{Et}_{3} \mathrm{~N}(300 \mu \mathrm{L})$ and $\mathrm{HCl}(1.00 \mathrm{~mL}, 1 \mathrm{M})$. The biphasic mixture was allowed to stir until two distinct layers were formed, and then the layers separated. The aqueous phase was extracted with $\mathrm{CH}_{2} \mathrm{Cl}_{2}(3 \times 5 \mathrm{~mL})$, and the combined organic layers were dried over $\mathrm{MgSO}_{4}$ and concentrated under reduced pressure to provide the crude product.

\section{General Procedure B (for Alkenes Prone to Acid-Promoted Polymerization)-}

To a flame-dried flask were added 2,3-butadienoate (1.00 equiv), $\mathrm{CH}_{2} \mathrm{Cl}_{2}(0.5 \mathrm{M})$, and alkene (2.00 equiv). $\mathrm{EtAlCl}_{2}$ (1.5 equiv, $1 \mathrm{M}$ in hexanes) was added down the walls of the flask. The reaction mixture was allowed to stir at room temperature for $6 \mathrm{~h}$ before carefully quenching with Rochelle's salt ( $5 \mathrm{~mL}$, sat.) to minimize polymerization of unreacted alkene starting material. The biphasic mixture was allowed to stir until two distinct layers were formed ( 10 $\mathrm{min})$, and then the layers were separated. The aqueous phase was extracted with $\mathrm{CH}_{2} \mathrm{Cl}_{2}(3 \times 5 \mathrm{~mL})$, and the combined organic layers were dried over $\mathrm{MgSO}_{4}$ and concentrated under reduced pressure to provide the crude product. 
Naphthalen-2-yl 2-(3-butylcyclobutylidene)acetate [3]-This compound was prepared according to General Procedure A utilizing naphthalen-2-yl buta-2,3-dienoate 2 and 1-hexene. Purification by silica gel chromatography (2\% EtOAc:hexanes) provided 42.5 $\mathrm{mg}(0.14 \mathrm{mmol}, 72 \%$ yield $)$ of the title compound as a bright yellow oil. $R_{f}=0.40(10 \%$ EtOAc:hexanes). IR (film): 3058 (w), 2956 (m), 2925 (m), 1735 (s), 1670 (m), 1511 (m), $1338(\mathrm{~m}) \mathrm{cm}^{-1} .{ }^{1} \mathrm{H}$ NMR $\left(600 \mathrm{MHz}, \mathrm{CDCl}_{3}\right) \delta 7.87-7.80(\mathrm{~m}, 2 \mathrm{H}), 7.78(\mathrm{~d}, J=7.9 \mathrm{~Hz}, 1 \mathrm{H})$, 7.59-7.54 (m, 1H), 7.50-7.40 (m, 2H), 7.27-7.21 (m, 1H), $5.88(\mathrm{t}, J=2.2 \mathrm{~Hz}, 1 \mathrm{H}), 3.40$ $3.30(\mathrm{~m}, 1 \mathrm{H}), 3.04-2.96(\mathrm{~m}, 1 \mathrm{H}), 2.81-2.73(\mathrm{~m}, 1 \mathrm{H}), 2.55-2.48(\mathrm{~m}, 1 \mathrm{H}), 2.45-2.39(\mathrm{~m}, 1 \mathrm{H})$, $1.56-1.49$ (m, 2H), 1.35-1.29 (m, 2H), 1.26 (tt, $J=11.9,6.7 \mathrm{~Hz}, 2 \mathrm{H}), 0.90$ (t, $J=7.2 \mathrm{~Hz}$, $3 \mathrm{H}) .{ }^{13} \mathrm{C}$ NMR $\left(100 \mathrm{MHz}, \mathrm{CDCl}_{3}\right) \delta 168.8,165.0,148.6,134.0,131.5,129.3,127.9,127.7$, 126.5, 125.6, 121.6, 118.7, 112.2, 40.0, 38.6, 36.3, 31.5, 29.7, 22.7, 14.2. HRMS (EI-MS): calcd for $\mathrm{C}_{20} \mathrm{H}_{22} \mathrm{O}_{2}[\mathrm{M}]^{+}$294.1614; found 294.1611.

Phenyl 2-Bromo-2-(3-butylcyclobutylidene)acetate [5]-This compound was prepared according to General Procedure A utilizing phenyl 2-bromobuta-2,3-dienoate $\mathbf{4}$ and 1-hexene. Purification by silica gel chromatography (2\% EtOAc:hexanes) provided $66.6 \mathrm{mg}$ $\left(0.17 \mathrm{mmol}, 87 \%\right.$ yield) of the title compound as a yellow oil. $R_{f}=0.40(10 \%$

EtOAc:hexanes). IR (film): 2956 (s), 2926 (s), 2871 (m), 2856 (m), 1738 (s), 1641 (m), 1593 (m), 1492 (s), 1337 (s), 1244 (s), 1162 (s), 1109 (m), 974 (m) cm ${ }^{-1} .{ }^{1} \mathrm{H}$ NMR (400 MHz, $\left.\mathrm{CDCl}_{3}\right) \delta 7.39(\mathrm{t}, J=7.9 \mathrm{~Hz}, 2 \mathrm{H}), 7.28-7.21(\mathrm{~m}, 1 \mathrm{H}), 7.13(\mathrm{dd}, J=7.9,1.6 \mathrm{~Hz}, 2 \mathrm{H}), 3.37-$ $3.21(\mathrm{~m}, 1 \mathrm{H}), 3.05-2.89(\mathrm{~m}, 1 \mathrm{H}), 2.73(\mathrm{ddd}, J=18.8,6.3,4.0 \mathrm{~Hz}, 1 \mathrm{H}), 2.53-2.30(\mathrm{~m}, 2 \mathrm{H})$, $1.53(\mathrm{q}, J=7.4 \mathrm{~Hz}, 2 \mathrm{H}), 1.41-1.21(\mathrm{~m}, 4 \mathrm{H}), 0.91(\mathrm{t}, J=7.0 \mathrm{~Hz}, 3 \mathrm{H}) .{ }^{13} \mathrm{C}$ NMR $(100 \mathrm{MHz}$, $\left.\mathrm{CDCl}_{3}\right) \delta 165.5,160.9,150.9,129.6,126.1,121.6,105.1,40.5,40.5,36.1,29.6,29.5,22.7$, 14.2. HRMS (EI-MS): calcd for $\mathrm{C}_{16} \mathrm{H}_{19} \mathrm{O}_{2} \mathrm{Br}_{1}[\mathrm{M}]^{+}$322.0563; found 322.0574 .

Phenyl 2-(3-Methyl-3-phenylcyclobutylidene)acetate [6]-This compound was prepared according to General Procedure A utilizing phenyl 2,3-butadienoate 1 and 1hexene. Purification by silica gel chromatography (2\% EtOAc:hexanes, gradient) provided $38.8 \mathrm{mg}(0.18 \mathrm{mmol}, 90 \%$ yield $)$ of the title compound as a pale yellow oil. $R_{f}=0.44$ (10\% EtOAc:hexanes). IR (film): 2956 (s), 2924 (s), 2853 (m), 1735 (s), 1671 (s), 1492 (s) $\mathrm{cm}^{-1} .{ }^{1} \mathrm{H}$ NMR $\left(400 \mathrm{MHz}, \mathrm{CDCl}_{3}\right) \delta$ 7.41-7.33 (m, 2H), 7.24-7.18 (m, 1H), 7.14-7.08 (m, $2 \mathrm{H}), 5.83(\mathrm{p}, J=2.2 \mathrm{~Hz}, 1 \mathrm{H}), 3.51-3.20(\mathrm{~m}, 1 \mathrm{H}), 3.06-2.92(\mathrm{~m}, 1 \mathrm{H}), 2.80-2.67(\mathrm{~m}, 1 \mathrm{H})$, 2.57-2.35 (m, 2H), 1.60-1.46 (m, 2H), 1.39-1.18 (m, 4H), $0.90(\mathrm{t}, J=7.1 \mathrm{~Hz}, 3 \mathrm{H}) .{ }^{13} \mathrm{C}$ NMR $\left(100 \mathrm{MHz}, \mathrm{CDCl}_{3}\right) \delta 168.5,164.9,150.9,129.4,125.6,121.9,112.2,39.9,38.6,36.3$, 31.5, 29.7, 22.7, 14.2. HRMS (EI-MS): calcd for $\mathrm{C}_{16} \mathrm{H}_{20} \mathrm{O}_{2}[\mathrm{M}]^{+}$244.1458; found 244.1448 .

Phenyl 2-(3-Benzylcyclobutylidene)acetate [7]-This compound was prepared according to General Procedure A utilizing phenyl 2,3-butadienoate $\mathbf{1}$ and allylbenzene. Purification by silica gel chromatography (2\% EtOAc:hexanes) provided $31.3 \mathrm{mg}(0.11$ mmol, 56\% yield) of the title compound as a clear colorless oil. $R_{f}=0.40(10 \%$ EtOAc:hexanes). IR (film): 3061 (m), 3028 (m), 2956 (m), 2909 (m), 1730 (s), 1672 (s), $1483(\mathrm{~s}) \mathrm{cm}^{-1} .{ }^{1} \mathrm{H}$ NMR $\left(400 \mathrm{MHz}, \mathrm{CDCl}_{3}\right) \delta$ 7.42-7.34 (m, 2H), 7.34-7.27 (m, 2H), 7.25$7.16(\mathrm{~m}, 4 \mathrm{H}), 7.14-7.08(\mathrm{~m}, 2 \mathrm{H}), 5.87(\mathrm{p}, J=2.2 \mathrm{~Hz}, 1 \mathrm{H}), 3.43-3.27(\mathrm{~m}, 1 \mathrm{H}), 3.05-2.95(\mathrm{~m}$, 1H), 2.95-2.72 (m, 4H), 2.69-2.60 (m, 1H). $\left.{ }^{13} \mathrm{C} \mathrm{NMR} \mathrm{(100} \mathrm{MHz,} \mathrm{CDCl}_{3}\right) \delta$ 167.3, 164.8, 
150.8, 140.3, 129.4, 128.7, 128.6, 126.3, 125.7, 121.9, 112.6, 42.3, 39.6, 38.2, 32.5. HRMS

(EI-MS): calcd for $\mathrm{C}_{19} \mathrm{H}_{18} \mathrm{O}_{2}[\mathrm{M}]^{+} 278.1301$; found 278.1291.

Phenyl 2-(3-(2-(4,4,5,5-Tetramethyl-1,3,2-dioxaborolan-2-yl)benzyl)cyclobutylidene)acetate [8]-Phenyl 2,3-butadienoate 1 (353 $\mu \mathrm{L}, 2.13 \mathrm{mmol}$, 1.00 equiv) was added to a $25 \mathrm{~mL}$ round-bottom flask, and the flask was evacuated and purged $3 \times$ with $\mathrm{N}_{2} . \mathrm{CH}_{2} \mathrm{Cl}_{2}(4.30 \mathrm{~mL}, 0.20 \mathrm{M})$ was added followed by 2-allylphenylboronic acid pinacol ester ( $1.04 \mathrm{~g}, 4.26 \mathrm{mmol}, 2.00$ equiv). The mixture was stirred at room temperature for $5 \mathrm{~min}$ before the addition of $\mathrm{EtAlCl}_{2}(3.20 \mathrm{~mL}, 3.20 \mathrm{mmol}, 1.50$ equiv). Upon complete addition of the Lewis acid, the reaction was allowed to stir at room temperature for an additional $16 \mathrm{~h}$. The reaction was then quenched with Rochelle's salt (10 $\mathrm{mL}$, sat.). The biphasic mixture was allowed to stir until two distinct layers were formed, and then the layers were separated. The aqueous phase was extracted with $\mathrm{CH}_{2} \mathrm{Cl}_{2}(3 \times 8$ $\mathrm{mL}$ ), and the combined organic layers were dried over $\mathrm{MgSO}_{4}$ and concentrated under reduced pressure to provide the crude product. Purification by silica gel chromatography (2$6 \%$ EtOAc:hexanes, gradient) provided $647 \mathrm{mg}$ ( $1.36 \mathrm{mmol}, 64 \%$ yield $)$ of the title compound as a clear colorless oil. $R_{f}=0.27$ (10\% EtOAc:hexanes). IR (film): 3480 (br), 2956 (m), 2909 (m), 1730 (s), 1643 (s), 1346 (w), 1277 (w), 1160 (w) cm ${ }^{-1} .{ }^{1} \mathrm{H}$ NMR (400 $\left.\mathrm{MHz}, \mathrm{CDCl}_{3}\right)(\mathrm{dd}, J=7.5,1.6 \mathrm{~Hz}, 1 \mathrm{H}), 7.40-7.30(\mathrm{~m}, 3 \mathrm{H}), 7.24-7.18(\mathrm{~m}, 2 \mathrm{H}), 7.16(\mathrm{~d}, J=$ $7.7 \mathrm{~Hz}, 1 \mathrm{H}), 7.12-7.08(\mathrm{~m}, 2 \mathrm{H}), 5.87-5.80(\mathrm{~m}, 1 \mathrm{H}), 3.35-3.22(\mathrm{~m}, 1 \mathrm{H}), 3.14$ (qd, $J=13.2$, $7.0 \mathrm{~Hz}, 2 \mathrm{H}), 2.96-2.82(\mathrm{~m}, 2 \mathrm{H}), 2.79-2.61(\mathrm{~m}, 2 \mathrm{H}), 1.35$ (s, 12H). ${ }^{13} \mathrm{C} \mathrm{NMR}(100 \mathrm{MHz}$, $\left.\mathrm{CDCl}_{3}\right) \delta 168.2,164.9,150.9,147.1,136.4,131.1,129.5,129.4,125.6,125.5,121.9,112.2$, 83.7, 41.6, 39.6, 38.1, 33.7, 25.0, 25.0. HRMS (EI-MS): calcd for $\mathrm{C}_{19} \mathrm{H}_{18} \mathrm{O}_{2}[\mathrm{M}]^{+}$278.1301; found 278.1291 .

\section{Phenyl 2-(3-((4,4,5,5-Tetramethyl-1,3,2-dioxaborolan-2-yl)- methyl)cyclobutylidene)acetate [9]—This compound was prepared according to} General Procedure B utilizing phenyl 2,3-butadienoate 1 and allylboronic acid pinacol ester. Purification by silica gel column chromatography (2-5\% EtOAc:hexanes, gradient) provided $48.6 \mathrm{mg}$ ( $0.15 \mathrm{mmol}, 74 \%$ yield $)$ of the title compound as a clear colorless oil. $R_{f}=0.35$ (10\% EtOAc:hexanes). IR (film): 3447 (br), 2978 (m), 1738 (s), 1669 (s), 1595 (m), 1494 (m), $1372(\mathrm{~s}), 1335(\mathrm{~m}) \mathrm{cm}^{-1} .{ }^{1} \mathrm{H}$ NMR (400 MHz, $\left.\mathrm{CDCl}_{3}\right) \delta$ 7.42-7.30 (m, 2H), 7.24-7.15 (m, 1H), 7.15-7.04 (m, 2H), $5.81(\mathrm{p}, J=2.2 \mathrm{~Hz}, 1 \mathrm{H}), 3.51-3.30(\mathrm{~m}, 1 \mathrm{H}), 3.14-2.96(\mathrm{~m}, 1 \mathrm{H})$, 2.79-2.70 (m, 1H), 2.68-2.59 (m, 1H), 2.57-2.47 (m, 1H), $1.23(\mathrm{~s}, 12 \mathrm{H}), 1.09$ (d, J= 7.6 $\mathrm{Hz}, 2 \mathrm{H}) .{ }^{13} \mathrm{C} \mathrm{NMR}\left(100 \mathrm{MHz}, \mathrm{CDCl}_{3}\right) \delta 168.5,165.0,150.9,129.4,125.6,121.9,111.9$, 83.3, 42.1, 40.8, 27.8, 25.0, 24.9. HRMS (CI-MS): calcd for $\mathrm{C}_{19} \mathrm{H}_{24} \mathrm{O}_{4} \mathrm{~B}_{1}[\mathrm{M}-$ $\mathrm{H}]^{+}$327.1762; found 327.1754 .

Phenyl 2-(3-(But-3-en-1-yl)cyclobutylidene)acetate [10]—This compound was prepared according to General Procedure B utilizing phenyl 2,3-butadienoate 1 and 1,5hexadiene. Purification by silica gel column chromatography (2-5\% EtOAc:hexanes, gradient) provided $35.6 \mathrm{mg}(0.15 \mathrm{mmol}, 74 \%$ yield $)$ of the title compound as a clear colorless oil. $R_{f}=0.39$ (10\% EtOAc:hexanes). IR (film): 3076 (w), 2924 (m), 1738 (s), 1669 (s), $1640(\mathrm{~m}), 1493(\mathrm{~m}), 1340(\mathrm{~m}), 913(\mathrm{~s}) \mathrm{cm}^{-1} .{ }^{1} \mathrm{H}$ NMR (500 MHz, $\left.\mathrm{CDCl}_{3}\right) \delta$ 7.41-7.31 (m, 2H), 7.25-7.16 (m, 1H), 7.15-7.06 (m, 2H), 5.89-5.74 (m, 2H), 5.02 (dq, $J=17.1,1.7$ 
$\mathrm{Hz}, 1 \mathrm{H}), 4.96$ (ddt, $J=10.2,2.3,1.2 \mathrm{~Hz}, 1 \mathrm{H}), 3.40-3.29(\mathrm{~m}, 1 \mathrm{H}), 3.05-2.95(\mathrm{~m}, 1 \mathrm{H}), 2.81-$ $2.71(\mathrm{~m}, 1 \mathrm{H}), 2.56-2.39(\mathrm{~m}, 2 \mathrm{H}), 2.09-2.01(\mathrm{~m}, 2 \mathrm{H}), 1.63(\mathrm{q}, J=7.5 \mathrm{~Hz}, 2 \mathrm{H}) .{ }^{13} \mathrm{C}$ NMR $\left(100 \mathrm{MHz}, \mathrm{CDCl}_{3}\right) \delta 168.03,164.86,150.85,138.41,129.43,125.63,121.89,114.86$, 112.29, 39.77, 38.45, 35.80, 31.78, 31.04. HRMS (EI-MS): calcd for $\mathrm{C}_{16} \mathrm{H}_{19} \mathrm{O}_{2}[\mathrm{M}+$ $\mathrm{H}]^{+}$243.1380; found 243.1383 .

Phenyl 2-(3-Cyclohexylcyclobutylidene)acetate [11]-This compound was prepared according to General Procedure A utilizing phenyl 2,3-butadienoate $\mathbf{1}$ and vinylcyclohexane. Purification by silica gel column chromatography (2\% EtOAc:hexanes) provided $30.0 \mathrm{mg}$ ( $0.17 \mathrm{mmol}, 84 \%$ yield, $13.6: 1 \mathrm{rr}$ ) of the title compound as a white solid. $R_{f}=0.49$ (10\% EtOAc:hexanes). IR (film): 2953 (m), 2849 (s), 1733 (s), 1672 (s), 1596 (m), 1493 (m), 1340 (m), 1163 (s), 1089 (m) 954 (m) cm ${ }^{-1} .{ }^{1} \mathrm{H} \mathrm{NMR}\left(400 \mathrm{MHz}, \mathrm{CDCl}_{3}\right) \delta 7.37$ (t, $J=7.9 \mathrm{~Hz}$, $2 \mathrm{H}), 7.21(\mathrm{t}, J=7.5 \mathrm{~Hz}, 1 \mathrm{H}), 7.11(\mathrm{dd}, J=7.7,1.6 \mathrm{~Hz}, 2 \mathrm{H}), 5.86-5.77(\mathrm{~m}, 1 \mathrm{H}), 3.36-3.20$ (m, 1H), 2.98-2.85 (m, 1H), 2.85-2.74 (m, 1H), 2.64-2.51 (m, 1H), 2.19-2.02 (m, 1H), 1.82-1.61 (m, 6H), 1.33-1.12 (m, 4H), 0.94-0.77 (m, 2H). $\left.{ }^{13} \mathrm{C} \mathrm{NMR} \mathrm{(125} \mathrm{MHz,} \mathrm{CDCl}_{3}\right) \delta$ 168.2, 164.9, 150.9, 129.4, 125.6, 121.9, 111.9, 44.0, 38.3, 37.4, 37.0, 30.2, 26.6, 26.2. HRMS (EI-MS): calcd for $\mathrm{C}_{18} \mathrm{H}_{23} \mathrm{O}_{2}[\mathrm{M}+\mathrm{H}]^{+} 271.1693$; found 271.1683. Mp 52-54 ${ }^{\circ} \mathrm{C}$

\section{Phenyl 2-(3-(((tert-Butyldimethylsilyl)oxy)methyl)-cyclobutylidene)acetate [12]}

-This compound was prepared according to General Procedure A utilizing phenyl 2,3butadienoate 1 and (allyloxy)(tert-butyl)dimethylsilane. Purification by silica gel column chromatography (hexanes to 3\% EtOAc:hexanes, gradient) provided $19.3 \mathrm{mg}(0.58,29 \%$ yield) of the title compound as a pale yellow oil. $R_{f}=0.28$ (10\% EtOAc:hexanes). IR (film): 2965 (s), 2932 (s), 2893 (m), 1740 (s), 1672 (m), 1493 (m), 1343 (m), $1192(\mathrm{~s}) \mathrm{cm}^{-1} .{ }^{1} \mathrm{H}$ NMR (400 MHz, $\left.\mathrm{CDCl}_{3}\right) \delta$ 7.42-7.31 (m, 2H), 7.25-7.18 (m, 1H), 7.13-7.07 (m, 2H), 5.88-5.82 (m, 1H), 3.73-3.60 (m, 2H), 3.31-3.15 (m, 1H), 3.01-2.84 (m, 2H), 2.79-2.57 $(\mathrm{m}, 2 \mathrm{H}), 0.95-0.87(\mathrm{~m}, 9 \mathrm{H}), 0.07(\mathrm{~d}, J=1.3 \mathrm{~Hz}, 6 \mathrm{H}) .{ }^{13} \mathrm{C} \mathrm{NMR}\left(100 \mathrm{MHz}, \mathrm{CDCl}_{3}\right) \delta$ 168.1, 164.9, 150.9, 129.4, 125.6, 121.9, 112.3, 65.9, 36.2, 34.9, 33.2, 26.1, 18.5, -5.18. HRMS (CI-MS): calcd for $\mathrm{C}_{19} \mathrm{H}_{29} \mathrm{O}_{3} \mathrm{Si}_{1}[\mathrm{M}+\mathrm{H}]^{+} 333.1880$; found 333.1888 .

\section{Phenyl 2-(3-(2-((tert-Butyldimethylsilyl)oxy)ethyl)-cyclobutylidene)acetate [13]}

-This compound was prepared according to General Procedure A utilizing phenyl 2,3butadienoate 1 and (but-3-en-1-yloxy)(tert-butyl)dimethylsilane. Purification by silica gel column chromatography (2\% EtOAc:hexanes) provided $43.7 \mathrm{mg}$ (63\% yield) of the title compound as a clear colorless oil. $R_{f}=0.44(0.13 \mathrm{mmol}, 10 \%$ EtOAc:hexanes). IR (film): 2964 (s), 2929 (s), 2898 (m), 1739 (s), $1672(\mathrm{~m}), 1493$ (m), 1339 (m), $1199(\mathrm{~s}) \mathrm{cm}^{-1} .{ }^{1} \mathrm{H}$ NMR (500 MHz, $\left.\mathrm{CDCl}_{3}\right) \delta 7.36(\mathrm{dd}, J=8.4,7.4 \mathrm{~Hz}, 2 \mathrm{H}), 7.22-7.17(\mathrm{~m}, 1 \mathrm{H}), 7.11-7.07(\mathrm{~m}$, $2 \mathrm{H}), 5.83(\mathrm{p}, J=2.4 \mathrm{~Hz}, 1 \mathrm{H}), 3.62(\mathrm{td}, J=6.3,1.7 \mathrm{~Hz}, 2 \mathrm{H}), 3.40-3.30(\mathrm{~m}, 1 \mathrm{H}), 3.06-2.96$ (m, 1H), 2.84-2.76 (m, 1H), 2.60-2.52 (m, 2H), $1.75(\mathrm{q}, J=6.5 \mathrm{~Hz}, 2 \mathrm{H}), 0.89(\mathrm{~s}, 9 \mathrm{H}), 0.04$ (s, 6H). ${ }^{13} \mathrm{C} \mathrm{NMR}\left(125 \mathrm{MHz}, \mathrm{CDCl}_{3}\right) \delta 168.26,164.85,150.87,129.43,125.62,121.89$, 112.24, 61.49, 39.83, 39.45, 38.57, 28.57, 26.09, 18.46, -5.21. HRMS (EI-MS): calcd for $\mathrm{C}_{16} \mathrm{H}_{21} \mathrm{O}_{3} \mathrm{Si}_{1}\left[\mathrm{M}-\mathrm{C}_{4} \mathrm{H}_{9}\right]^{+}$289.1254; found 289.1259.

Phenyl 2-(3-Phenylcyclobutylidene)acetate [14]—Phenyl 2,3-butadienoate 1 (1.00 g, $6.55 \mathrm{mmol}, 1.00$ equiv) was added to a $100 \mathrm{~mL}$ flame-dried round-bottom flask and purged 
$3 \times$ with $\mathrm{N}_{2} . \mathrm{CH}_{2} \mathrm{C}_{12}(33.0 \mathrm{~mL}, 0.20 \mathrm{M})$ was added followed by styrene $(1.50 \mathrm{~mL}, 13.1$ mmol, 2.00 equiv), and the resulting mixture was allowed to stir for $5 \mathrm{~min}$. $\mathrm{EtAlCl}_{2}(9.80$ $\mathrm{mL}, 9.80 \mathrm{mmol}, 1.50$ equiv) was added, and the reaction was allowed to stir for $6 \mathrm{~h}$ at room temperature. After $6 \mathrm{~h}$, the reaction was quenched with Rochelle's salt $(10 \mathrm{~mL})$ and extracted with $\mathrm{CH}_{2} \mathrm{Cl}_{2}(3 \times 10 \mathrm{~mL})$. The combined organic phases were washed with brine, dried over $\mathrm{MgSO}_{4}$, filtered, and concentrated to yield a crude yellow residue. Purification by silica gel column chromatography (2-4\% EtOAc:hexanes, gradient) provided $1.48 \mathrm{~g}$ (5.60 mmol, $90 \%$ yield) of the title compound as a pale yellow oil. $R_{f}=0.39(10 \%$

EtOAc:hexanes). IR (film): 3040 (w), 2965 (w), 2934 (m), 2103 (w), 1643 (s), 1492 (m), 1400 (w), 1247 (m), 1164 (m), $1090(\mathrm{w}) \mathrm{cm}^{-1} .{ }^{1} \mathrm{H}$ NMR (400 MHz, $\left.\mathrm{CDCl}_{3}\right) \delta$ 7.43-7.31 (m, 4H), 7.31-7.27 (m, 2H), 7.25-7.19 (m, 2H), 7.15-7.09 (m, 2H), 6.07-5.68 (m, 1H), 3.79-3.64 (m, 2H), 3.42-3.24 (m, 2H), 3.13-3.01 (m, 1H). $\left.{ }^{13} \mathrm{C} \mathrm{NMR} \mathrm{(125} \mathrm{MHz,} \mathrm{CDCl}_{3}\right) \delta$ 166.1, 164.8, 150.8, 144.5, 129.5, 128.7, 126.6, 126.5, 125.7, 121.9, 112.5, 41.6, 40.5, 35.8. HRMS (EI-MS): calcd for $\mathrm{C}_{18} \mathrm{H}_{17} \mathrm{O}_{2}[\mathrm{M}+\mathrm{H}]^{+}$265.1223; found 265.1212.

Phenyl 2-(3-(4-Fluorophenyl)cyclobutylidene)acetate [15]-This compound was prepared according to General Procedure B utilizing phenyl 2,3-butadienoate 1 and 4fluorostyrene. Purification by silica gel column chromatography (2\% EtOAc:hexanes) provided $34.4 \mathrm{mg}(0.12 \mathrm{mmol}, 61 \%$ yield $)$ of the title compound as a pale yellow oil. $R_{f}=$ 0.42 (10\% EtOAc:hexanes). IR (film): 2965 (w), 2934 (m), 1731 (s), 1672 (s), $1492(\mathrm{~m})$, $1341(\mathrm{~s}), 1163(\mathrm{~s}) \mathrm{cm}^{-1} .{ }^{1} \mathrm{H}$ NMR $\left(400 \mathrm{MHz}, \mathrm{CDCl}_{3}\right) \delta$ 7.42-7.35 (m, 2H), 7.33-7.28 (m, 2H), 7.25-7.19 (m, 3H), 7.14-7.10 (m, 2H), 5.95 (p, $J=2.2 \mathrm{~Hz}, 1 \mathrm{H}), 3.79-3.60$ (m, 2H), 3.41-3.30 (m, 1H), 3.30-3.18 (m, 1H), 3.09-2.95 (m, 1H). $\left.{ }^{13} \mathrm{C} \mathrm{NMR} \mathrm{(100} \mathrm{MHz,} \mathrm{CDCl}_{3}\right) \delta$ 165.59, 164.72, 161.61 (d, $J=244.4 \mathrm{~Hz}), 150.77,140.20,129.49,127.95$ (d, $J=8.0 \mathrm{~Hz})$, $125.75,121.84,115.42$ (d, $J=21.2 \mathrm{~Hz}) .112 .64,41.75,40.63,35.15^{19} \mathrm{~F}$ NMR $(375 \mathrm{MHz}$, $\mathrm{CDCl}_{3}$ ) $\delta-116.70$ (q, $J=6.4,5.2 \mathrm{~Hz}$ ). HRMS (EI-MS): calcd for $\mathrm{C}_{18} \mathrm{H}_{16} \mathrm{O}_{2} \mathrm{~F}_{1}[\mathrm{M}+$ $\mathrm{H}]^{+}$283.1129; found 283.1129 .

Phenyl 2-(3-(4-Bromophenyl)cyclobutylidene)acetate (16)-This compound was prepared according to General Procedure B utilizing phenyl 2,3-butadienoate 1 and 4bromostyrene 39. Purification by silica gel column chromatography (2\% EtOAc:hexanes) provided $59.7 \mathrm{mg}(0.14 \mathrm{mmol}, 69 \%$ yield $)$ of the title compound as a clear colorless oil. $R_{f}=$ 0.42 (10\% EtOAc:hexanes). IR (film): 3042 (w), 2960 (m), 2920 (m), 1723 (s), 1676 (m), $1490(\mathrm{~s}), 1341(\mathrm{~s}) \mathrm{cm}^{-1} .{ }^{1} \mathrm{H}$ NMR (400 MHz, $\left.\mathrm{CDCl}_{3}\right) \delta$ 7.49-7.42 (m, 2H), 7.42-7.35 (m, $2 \mathrm{H}), 7.25-7.20(\mathrm{~m}, 1 \mathrm{H}), 7.19-7.08(\mathrm{~m}, 4 \mathrm{H}), 5.95(\mathrm{p}, J=2.1 \mathrm{~Hz}, 1 \mathrm{H}), 3.81-3.58(\mathrm{~m}, 2 \mathrm{H})$, 3.43-3.19 (m, 2H), 3.09-2.95 (m, 1H). ${ }^{13} \mathrm{C}$ NMR (100 MHz, $\left.\mathrm{CDCl}_{3}\right) \delta$ 165.1, 164.5, 150.6, 143.35, 131.6, 129.3, 128.1, 125.6, 121.7, 120.1, 112.6, 41.3, 40.2, 35.1. HRMS (EI-MS): calcd for $\mathrm{C}_{18} \mathrm{H}_{15} \mathrm{O}_{2} \mathrm{Br}_{1}[\mathrm{M}]^{+} 342.0250$; found 342.0241 .

\section{Phenyl 2-(3-(4-(4,4,5,5-Tetramethyl-1,3,2-dioxaborolan-2-yl)-} phenyl)cyclobutylidene)acetate [17]—This compound was prepared according to General Procedure B utilizing phenyl 2,3-butadienoate $\mathbf{1}$ and 4-vinylphenylboronic acid pinacol ester 44. Purification by silica gel column chromatography (2-10\% EtOAc:hexanes, gradient) provided $29.6 \mathrm{mg}$ ( $0.08 \mathrm{mmol}, 38 \%$ yield) of the title compound as a pale yellow oil. $R_{f}=0.46$ (10\% EtOAc:hexanes). IR (film): 3447 (br), 2977 (m), 2929 (m), 1731 (s), 
$1675(\mathrm{~m}), 1612(\mathrm{~m}), 1493(\mathrm{~m}), 1320(\mathrm{~m}), 1088(\mathrm{~s}) \mathrm{cm}^{-1} .{ }^{1} \mathrm{H}$ NMR $\left(600 \mathrm{MHz}, \mathrm{CDCl}_{3}\right) \delta$ $7.80(\mathrm{~d}, J=7.7 \mathrm{~Hz}, 2 \mathrm{H}), 7.38(\mathrm{t}, J=7.8 \mathrm{~Hz}, 2 \mathrm{H}), 7.30(\mathrm{~d}, J=7.7 \mathrm{~Hz}, 2 \mathrm{H}), 7.22(\mathrm{t}, J=7.5$ $\mathrm{Hz}, 1 \mathrm{H}), 7.15-7.10(\mathrm{~m}, 2 \mathrm{H}), 5.96-5.92(\mathrm{~m}, 1 \mathrm{H}), 3.82-3.63(\mathrm{~m}, 2 \mathrm{H}), 3.39-3.24(\mathrm{~m}, 2 \mathrm{H})$, 3.13-3.03 (m, 1H), $1.35(\mathrm{~s}, 12 \mathrm{H}) .{ }^{13} \mathrm{C}$ NMR $\left(100 \mathrm{MHz}, \mathrm{CDCl}_{3}\right) \delta 165.9,164.8,150.8$, $147.8,135.2,129.5,125.9,125.7,121.9,112.6,83.9,41.5,40.4,35.9,25.0$. HRMS (ESITOF): calcd for $\mathrm{C}_{24} \mathrm{H}_{27} \mathrm{O}_{4} \mathrm{~B}_{1} \mathrm{Na}_{1}[\mathrm{M}+\mathrm{Na}]^{+} 413.1900$; found 413.1884 .

Phenyl 2-(3-(4-Acetoxyphenyl)cyclobutylidene)acetate [18]-This compound was prepared according to General Procedure B utilizing phenyl 2,3-butadienoate 1 and 4acetoxystyrene with increased reaction time $(16 \mathrm{~h})$. Purification by silica gel column chromatography (5-20\% EtOAc:hexanes, gradient) provided $42.7 \mathrm{mg}(0.13 \mathrm{mmol}, 66 \%$ yield) of the title compound as a pale yellow oil. $R_{f}=0.23$ (20\% EtOAc:hexanes). IR (film): 3042 (w), 2923 (w), 1763 (s), 1734 (s), 1674 (m), 1508 (s), 1342 (m), 1090 (m) cm ${ }^{-1} .{ }^{1} \mathrm{H}$ NMR (400 MHz, $\left.\mathrm{CDCl}_{3}\right) \delta$ 7.42-7.34 (m, 2H), 7.32-7.27 (m, 2H), 7.25-7.19 (m, 1H), 7.15-7.11 (m, 2H), 7.09-7.02 (m, 2H), 5.95 (p, $J=2.2 \mathrm{~Hz}, 1 \mathrm{H}), 3.80-3.62(\mathrm{~m}, 2 \mathrm{H}), 3.43-$ $3.21(\mathrm{~m}, 2 \mathrm{H}), 3.12-2.97(\mathrm{~m}, 1 \mathrm{H}), 2.30(\mathrm{~s}, 3 \mathrm{H}) .{ }^{13} \mathrm{C} \mathrm{NMR}\left(100 \mathrm{MHz}, \mathrm{CDCl}_{3}\right) \delta$ 169.8, 165.7, 164.7, 150.8, 149.2, 142.1, 129.7, 129.5, 127.5, 125.7, 121.8, 121.7, 121.4, 112.6, 41.6, 40.5, 35.3, 21.3. Mp 67-69 ${ }^{\circ} \mathrm{C}$. HRMS (CI-MS): calcd for $\mathrm{C}_{20} \mathrm{H}_{18} \mathrm{O}_{4}[\mathrm{M}+\mathrm{H}]^{+} 323.1278$; found 323.1271 .

Phenyl 2-(3-(4-(Tosyloxy)phenyl)cyclobutylidene)acetate [19]-This compound was prepared according to General Procedure B utilizing phenyl 2,3-butadienoate 1 and 4tosylstyrene 41. Purification by silica gel column chromatography (5-25\% EtOAc:hexanes) provided $59.7 \mathrm{mg}\left(0.14 \mathrm{mmol}, 69 \%\right.$ yield) of the title compound as a white solid. $R_{f}=0.24$ (10\% EtOAc:hexanes) IR (film): 2924 (w), 1724 (s), 1676 (m), 1595 (m), 1503 (s), 1372 (s),

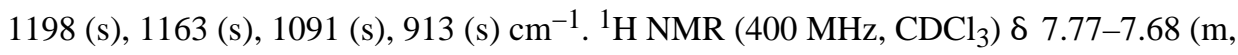
2H), 7.42-7.34 (m, 2H), 7.34-7.29 (m, 2H), 7.25-7.15 (m, 3H), 7.14-7.08 (m, 2H), 6.97$6.91(\mathrm{~m}, 2 \mathrm{H}), 5.93(\mathrm{p}, J=2.2 \mathrm{~Hz}, 1 \mathrm{H}), 3.76-3.59(\mathrm{~m}, 2 \mathrm{H}), 3.33$ (ddtd, $J=17.4,7.8,4.0,1.7$ $\mathrm{Hz}, 1 \mathrm{H}), 3.28-3.16(\mathrm{~m}, 1 \mathrm{H}), 3.07-2.92(\mathrm{~m}, 1 \mathrm{H}), 2.45$ (s, 3H). ${ }^{13} \mathrm{C} \mathrm{NMR}\left(100 \mathrm{MHz}, \mathrm{CDCl}_{3}\right)$ б 165.1, 164.5, 150.6, 148.0, 145.3, 143.4, 132.5, 129.7, 129.3, 128.5, 127.5, 125.6, 122.4, 121.7, 112.6, 41.4, 40.2, 35.1, 21.7. Mp 121-123 ${ }^{\circ} \mathrm{C}$. HRMS (EI-MS): calcd for $\mathrm{C}_{25} \mathrm{H}_{22} \mathrm{O}_{5} \mathrm{~S}_{1}[\mathrm{M}]^{+} 434.1182$; found 434.1197.

Phenyl 2-(3-(0-Tolyl)cyclobutylidene)acetate [20]-This compound was prepared according to General Procedure B utilizing phenyl 2,3-butadienoate 1 and 2-methylstyrene. Purification by silica gel column chromatography (2\% EtOAc:hexanes) provided $30.1 \mathrm{mg}$ ( $0.12 \mathrm{mmol}, 62 \%$ yield) of the title compound as a pale yellow oil. $R_{f}=0.40(10 \%$ EtOAc:hexanes). IR (film): 3062 (w), 2956 (w), 2921 (w), 1730 (s), 1676 (s), 1492 (s), 1342 (s), $1198(\mathrm{~s}) \mathrm{cm}^{-1} .{ }^{1} \mathrm{H}$ NMR $\left(400 \mathrm{MHz}, \mathrm{CDCl}_{3}\right) \delta 7.31(\mathrm{t}, J=7.7 \mathrm{~Hz}, 2 \mathrm{H}), 7.22(\mathrm{~d}, J=7.6$ $\mathrm{Hz}, 1 \mathrm{H}), 7.19-7.12(\mathrm{~m}, 2 \mathrm{H}), 7.11-7.03(\mathrm{~m}, 4 \mathrm{H}), 5.90-5.84(\mathrm{~m}, 1 \mathrm{H}), 3.81-3.69(\mathrm{~m}, 1 \mathrm{H})$, 3.69-3.58 (m, 1H), 3.22 (dddd, $J=19.3,11.4,8.3,3.8 \mathrm{~Hz}, 2 \mathrm{H}), 3.06-2.94(\mathrm{~m}, 1 \mathrm{H}), 2.21$ (s, $3 \mathrm{H}) .{ }^{13} \mathrm{C}$ NMR $\left(100 \mathrm{MHz}, \mathrm{CDCl}_{3}\right) \delta 165.9,164.9,150.8,141.6,136.1,130.4,129.5,126.6$, 126.2, 125.7, 125.0, 121.9, 112.1, 40.3, 39.1, 33.6, 19.8. HRMS (EI-MS): calcd for $\mathrm{C}_{19} \mathrm{H}_{18} \mathrm{O}_{2}[\mathrm{M}]^{+}$278.1301; found 278.1298. 
Phenyl 2-(3-(2-Bromophenyl)cyclobutylidene)acetate [21]-This compound was prepared according to General Procedure B utilizing phenyl 2,3-butadienoate 1 and 2bromostyrene 37. Purification by silica gel column chromatography (2\% EtOAc:hexanes) provided $64.3 \mathrm{mg}(0.19 \mathrm{mmol}, 93 \%$ yield $)$ of the title compound as a clear colorless oil. $R_{f}=$ 0.42 (10\% EtOAc:hexanes). IR (film): 3063 (w), 2970 (w), 2921 (w), 1727 (s), 1676 (s), 1594 (m), 1493 (s), 1164 (s), 1090 (s) cm ${ }^{-1} .{ }^{1} \mathrm{H}$ NMR (400 MHz, $\left.\mathrm{CDCl}_{3}\right) \delta 7.56$ (dd, $J=$ 8.0, 1.2 Hz, 1H), 7.46-7.26 (m, 4H), 7.28-7.16 (m, 1H), 7.17-7.03 (m, 3H), 5.94 (p, $J=2.3$ $\mathrm{Hz}, 1 \mathrm{H}), 4.05-3.89(\mathrm{~m}, 1 \mathrm{H}), 3.83-3.67(\mathrm{~m}, 1 \mathrm{H}), 3.47-3.35(\mathrm{~m}, 1 \mathrm{H}), 3.36-3.22(\mathrm{~m}, 1 \mathrm{H}), 3.01$ (dddd, $J=17.0,7.9,3.4,2.3 \mathrm{~Hz}, 1 \mathrm{H}) .{ }^{13} \mathrm{C}$ NMR $\left(100 \mathrm{MHz}, \mathrm{CDCl}_{3}\right) \delta 165.1,164.72,150.8$, 142.6, 133.1, 129.5, 128.1, 127.6, 127.0, 125.7, 124.3, 121.9, 112.5, 39.9, 39.3, 36.1. HRMS (CI-MS): calcd for $\mathrm{C}_{18} \mathrm{H}_{16} \mathrm{O}_{2} \mathrm{Br}_{1}[\mathrm{M}+\mathrm{H}]^{+}$343.0328; found 343.0328.

\section{Phenyl 2-(3-(2-(4,4,5,5-Tetramethyl-1,3,2-dioxaborolan-2-yl)-} phenyl)cyclobutylidene)acetate [22] - This compound was prepared according to General Procedure B utilizing phenyl 2,3-butadienoate 1 and 2-vinylphenylboronic acid pinacol ester. Purification by silica gel column chromatography (2-10\% EtOAc:hexanes, gradient) provided $28.9 \mathrm{mg}$ ( $0.07 \mathrm{mmol}, 37 \%$ yield $)$ of the title compound as a pale yellow oil. $R_{f}=0.46$ (10\% EtOAc:hexanes) IR (film): 3442 (br), 2976 (m), 2930 (w), 1732 (s), $1678(\mathrm{~m}), 1487(\mathrm{~m}), 1345(\mathrm{~s}), 1096(\mathrm{~m}) \mathrm{cm}^{-1} .{ }^{1} \mathrm{H} \mathrm{NMR}\left(400 \mathrm{MHz}, \mathrm{CDCl}_{3}\right) \delta 7.82$ (dd, $J=$ 7.4, 1.5 Hz, 1H), 7.52-7.31 (m, 4H), 7.31-7.17 (m, 2H), 7.17-7.07 (m, 2H), 6.04-5.83 (m, $1 \mathrm{H}), 4.39(\mathrm{p}, J=8.3 \mathrm{~Hz}, 1 \mathrm{H}), 3.80-3.58(\mathrm{~m}, 1 \mathrm{H}), 3.44-3.19(\mathrm{~m}, 2 \mathrm{H}), 3.12-2.92(\mathrm{~m}, 1 \mathrm{H})$, 1.35 (s, 12H). ${ }^{13} \mathrm{C}$ NMR $\left(100 \mathrm{MHz}, \mathrm{CDCl}_{3}\right) \delta 167.4,164.9,150.9,150.1,136.4,131.4$, 129.4, 125.7, 125.6, 124.8, 121.9, 112.1, 83.7, 41.3, 40.6, 34.8, 25.0, 25.0. HRMS (CI-MS): calcd for $\mathrm{C}_{24} \mathrm{H}_{27} \mathrm{O}_{4} \mathrm{~B}_{1}[\mathrm{M}]^{+} 390.1997$; found 390.2000 .

Phenyl 2-(3-(3-Bromophenyl)cyclobutylidene)acetate [23]-This compound was prepared according to General Procedure B utilizing phenyl 2,3-butadienoate 1 and 3bromostyrene 38. Purification by silica gel column chromatography (2\% EtOAc:hexanes) provided $53.5 \mathrm{mg}(0.16 \mathrm{mmol}, 78 \%$ yield $)$ of the title compound as a clear colorless oil. $R_{f}=$ 0.41 (10\% EtOAc:hexanes). IR (film): 3062 (w), 2924 (w), 1728 (s), 1678 (m), 1597 (m), $1492(\mathrm{~m}), 1163(\mathrm{~s}), 1088(\mathrm{~s}) \mathrm{cm}^{-1} .{ }^{1} \mathrm{H}$ NMR $\left(500 \mathrm{MHz}, \mathrm{CDCl}_{3}\right) \delta 7.45-7.41(\mathrm{~m}, 1 \mathrm{H}), 7.42-$ $7.34(\mathrm{~m}, 3 \mathrm{H}), 7.25-7.19(\mathrm{~m}, 3 \mathrm{H}), 7.15-7.09(\mathrm{~m}, 2 \mathrm{H}), 5.95(\mathrm{q}, J=2.2 \mathrm{~Hz}, 1 \mathrm{H}), 3.78-3.63(\mathrm{~m}$, 2H), 3.40-3.21 (m, 2H), 3.08-3.00 (m, 1H). ${ }^{13} \mathrm{C}$ NMR (125 MHz, $\left.\mathrm{CDCl}_{3}\right) \delta$ 165.0, 164.6, 150.8, 146.9, 130.3, 129.7, 129.7, 129.5, 125.8, 125.2, 122.8, 121.8, 112.9, 41.4, 40.3, 35.5. HRMS (CI-MS): calcd for $\mathrm{C}_{18} \mathrm{H}_{16} \mathrm{O}_{2} \mathrm{Br}_{1}[\mathrm{M}+\mathrm{H}]^{+}$343.0328; found 343.0325.

\section{Phenyl 2-(3-(3-(4,4,5,5-Tetramethyl-1,3,2-dioxaborolan-2-yl)-} phenyl)cyclobutylidene)acetate [24]-This compound was prepared according to General Procedure B utilizing phenyl 2,3-butadienoate 1 and 3-vinylphenylboronic acid pinacol ester 43. Purification by silica gel column chromatography (2-10\% EtOAc:hexanes gradient) provided $54.6 \mathrm{mg}(0.14 \mathrm{mmol}, 70 \%$ yield $)$ of the title compound as a clear colorless oil. $R_{f}=0.33$ (10\% EtOAc:hexanes) IR (film): 3448 (br), 2978 (m), 2930 (w), $1729(\mathrm{~s}), 1675(\mathrm{~m}), 1492(\mathrm{~m}), 1341(\mathrm{~s}), 1090(\mathrm{~m}) \mathrm{cm}^{-1} .{ }^{1} \mathrm{H} \mathrm{NMR}\left(500 \mathrm{MHz}, \mathrm{CDCl}_{3}\right) \delta 7.73$ (s, 1H), 7.71-7.67 (m, 1H), 7.43-7.34 (m, 4H), 7.22 (t, $J=7.4 \mathrm{~Hz}, 1 \mathrm{H}), 7.16-7.11(\mathrm{~m}, 2 \mathrm{H})$, 5.94 (p, $J=2.4 \mathrm{~Hz}, 1 \mathrm{H}$ ), 3.79-3.65 (m, 2H), 3.41-3.25 (m, 2H), 3.11 (ddt, $J=18.5,7.5,2.6$ 
$\mathrm{Hz}, 1 \mathrm{H}), 1.36(\mathrm{~s}, 12 \mathrm{H}) .{ }^{13} \mathrm{C} \mathrm{NMR}\left(125 \mathrm{MHz}, \mathrm{CDCl}_{3}\right) \delta 166.1,164.7,150.7,143.6,132.9$, $132.7,129.3,129.3,128.0,125.6,121.8,112.3,83.9,41.5,40.4,35.6,24.9$. HRMS (EIMS): calcd for $\mathrm{C}_{24} \mathrm{H}_{27} \mathrm{O}_{4} \mathrm{~B}_{1}[\mathrm{M}]^{+}$390.1997; found 390.1998 .

Phenyl 2-(3-Isopropyl-3-methylcyclobutylidene)acetate [25]—This compound was prepared according to General Procedure A utilizing phenyl 2,3-butadienoate 1 and 2,3dimethylbut-1-ene. Purification by silica gel column chromatography (2\% EtOAc:hexanes, gradient) provided $39.0 \mathrm{mg}(0.16 \mathrm{mmol}, 80 \%$ yield $)$ of the title compound as a pale yellow oil. $\mathrm{R}_{f}=0.40$ (10\% EtOAc:hexanes). IR (neat): 2959 (s), 2873 (m), 1722 (s), 1673 (s), 1595 (s), 1339 (s), $1248(\mathrm{~m}), 960(\mathrm{~m}) \mathrm{cm}^{-1} .{ }^{1} \mathrm{H}$ NMR $\left(400 \mathrm{MHz}, \mathrm{CDCl}_{3}\right) \delta$ 7.42-7.34 (m, 2H), 7.24-7.18 (m, 1H), 7.14-7.08 (m, 2H), 5.89 (p, $J=2.3 \mathrm{~Hz}, 1 \mathrm{H}), 2.99-2.89(\mathrm{~m}, 1 \mathrm{H}), 2.89$ $2.78(\mathrm{~m}, 1 \mathrm{H}), 2.74-2.62(\mathrm{~m}, 1 \mathrm{H}), 2.55-2.44(\mathrm{~m}, 1 \mathrm{H}), 1.75$ (hept, $J=6.8 \mathrm{~Hz}, 1 \mathrm{H}), 1.07$ (s, $3 \mathrm{H}), 0.86(\mathrm{~d}, J=6.8 \mathrm{~Hz}, 6 \mathrm{H}) .{ }^{13} \mathrm{C} \mathrm{NMR}\left(100 \mathrm{MHz}, \mathrm{CDCl}_{3}\right) \delta 166.6,164.9,150.9,129.4$, 125.6, 121.9, 113.4, 45.3, 43.9, 39.6, 37.0, 20.2, 17.0. HRMS (EI-MS): calcd for $\mathrm{C}_{16} \mathrm{H}_{20} \mathrm{O}_{2}$ $[\mathrm{M}]^{+}$244.1458; found 244.1450.

\section{Phenyl 2-(3-Methyl-3-((4,4,5,5-tetramethyl-1,3,2-dioxaborolan-2-} yl)methyl)cyclobutylidene)acetate (26)—This compound was prepared according to General Procedure B utilizing phenyl 2,3-butadienoate $\mathbf{1}$ and methallyl boronic acid pinacol ester. Purification by silica gel column chromatography (2-10\% EtOAc:hexanes, gradient) provided $49.7 \mathrm{mg}(0.15 \mathrm{mmol}, 73 \%$ yield $)$ of the title compound as a clear colorless oil. $R_{f}=$ 0.42 (10\% EtOAc:hexanes). IR (film): 3447 (br), 2978 (m), 2950 (m), 1738 (s), 1672 (s), $1493(\mathrm{~m}), 1359(\mathrm{~s}), 1274(\mathrm{~m}), 1144(\mathrm{~m}) \mathrm{cm}^{-1} .{ }^{1} \mathrm{H} \mathrm{NMR}\left(400 \mathrm{MHz}, \mathrm{CDCl}_{3}\right) \delta$ 7.40-7.33 (m, 2H), 7.23-7.17 (m, 1H), 7.12-7.07 (m, 2H), 5.87 (p, $J=2.3 \mathrm{~Hz}, 1 \mathrm{H}), 3.07-2.99(\mathrm{~m}, 1 \mathrm{H})$, 2.99-2.90 (m, 1H), 2.82-2.74 (m, 1H), 2.68-2.59 (m, 1H), 1.28-1.21 (m, 15H), $1.13(\mathrm{~s}$, 2H). ${ }^{13} \mathrm{C} \mathrm{NMR}\left(100 \mathrm{MHz}, \mathrm{CDCl}_{3}\right) \delta 167.2,164.7,150.7,129.2,125.4,121.8,113.0,83.0$, 47.7, 46.4, 34.1, 28.6, 24.8. HRMS (EI-MS): calcd for $\mathrm{C}_{20} \mathrm{H}_{27} \mathrm{O}_{4} \mathrm{~B}_{1}[\mathrm{M}]^{+} 342.1997$; found 342.1994 .

\section{Phenyl 2-(3-Phenyl-3-(4,4,5,5-tetramethyl-1,3,2-dioxaborolan-2-} yl)cyclobutylidene)acetate [27]—This compound was prepared according to General Procedure B utilizing phenyl 2,3-butadienoate 1 and 1-phenylvinylboronic acid pinacol ester 45. Purification by silica gel column chromatography (2-10\% EtOAc:hexanes gradient) provided $62.5 \mathrm{mg}(0.16 \mathrm{mmol}, 80 \%$ yield $)$ of the title compound as a clear colorless oil. $R_{f}=$ 0.4 (10\% EtOAc:hexanes). IR (film): 3428 (br), 3061 (w), 2978 (m), 2931 (w), 1728 (s), 1674 (s), 1493 (m), 1356 (s), 1319 (s), 1139 (m), 1094 (s) cm ${ }^{-1} .{ }^{1} \mathrm{H}$ NMR (500 MHz, $\left.\mathrm{CDCl}_{3}\right) \delta 7.38(\mathrm{dd}, J=8.5,7.4 \mathrm{~Hz}, 2 \mathrm{H}), 7.34-7.29(\mathrm{~m}, 2 \mathrm{H}), 7.24-7.19(\mathrm{~m}, 3 \mathrm{H}), 7.19-7.15$ (m, 1H), 7.14-7.10 (m, 2H), $5.86(\mathrm{p}, J=2.3 \mathrm{~Hz}, 1 \mathrm{H}), 3.84(\mathrm{dq}, J=17.6,2.8 \mathrm{~Hz}, 1 \mathrm{H}), 3.55-$ $3.41(\mathrm{~m}, 2 \mathrm{H}), 3.18$ (ddd, $J=16.8,3.6,2.3 \mathrm{~Hz}, 1 \mathrm{H}), 1.18(\mathrm{~s}, 12 \mathrm{H}) .{ }^{13} \mathrm{C}$ NMR $(125 \mathrm{MHz}$, $\left.\mathrm{CDCl}_{3}\right) \delta 166.4,164.7,150.9,146.6,129.4,128.4,126.1,125.6,125.3,121.9,112.2,84.1$, 43.4, 42.1, 24.6. HRMS (CI-MS): calcd for $\mathrm{C}_{26} \mathrm{H}_{32} \mathrm{O}_{4} \mathrm{~B}_{1}\left[\mathrm{M}+\mathrm{C}_{2} \mathrm{H}_{5}\right]^{+} 419.2388$; found 419.2383.

Phenyl 2-(3-Methyl-3-phenylcyclobutylidene)acetate [28]-This compound was prepared according to General Procedure A utilizing phenyl 2,3-butadienoate 1 and a- 
methylstyrene. Purification by silica gel column chromatography (2\% EtOAc:hexanes) provided $54.5 \mathrm{mg}(0.19 \mathrm{mmol}, 98 \%$ yield $)$ of the title compound as a pale yellow oil. $R_{f}=$ 0.44 (10\% EtOAc:hexanes). IR (film): 3059 (w), 3024 (w), 2958 (m), 2920 (w), 1729 (s), $1676(\mathrm{~m}), 1493(\mathrm{~s}), 1340(\mathrm{~s}), 1198(\mathrm{~s}), 1080(\mathrm{~m}) \mathrm{cm}^{-1} .{ }^{1} \mathrm{H} \mathrm{NMR}\left(600 \mathrm{MHz}, \mathrm{CDCl}_{3}\right) \delta 7.39$ $7.31(\mathrm{~m}, 3 \mathrm{H}), 7.26-7.18(\mathrm{~m}, 5 \mathrm{H}), 7.12-7.09(\mathrm{~m}, 2 \mathrm{H}), 5.95(\mathrm{p}, J=2.3 \mathrm{~Hz}, 1 \mathrm{H}), 3.53-3.46(\mathrm{~m}$, $1 \mathrm{H}), 3.39-3.32(\mathrm{~m}, 1 \mathrm{H}), 3.31-3.25(\mathrm{~m}, 1 \mathrm{H}), 3.00-2.93(\mathrm{~m}, 1 \mathrm{H}), 1.52(\mathrm{~s}, 3 \mathrm{H}) .{ }^{13} \mathrm{C}$ NMR $(100$ $\left.\mathrm{MHz}, \mathrm{CDCl}_{3}\right) \delta 165.1,164.8,150.8,149.6,129.5,128.6,126.0,125.7,125.2,121.9,113.6$, 46.8, 45.7, 40.2, 31.0 HRMS (EI-MS): calcd for $\mathrm{C}_{19} \mathrm{H}_{18} \mathrm{O}_{2}[\mathrm{M}]^{+} 278.1301$; found 278.1310 .

Phenyl 2-(3,3-Diphenylcyclobutylidene)acetate [29]—This compound was prepared according to General Procedure B utilizing phenyl 2,3-butadienoate 1 and 1,1diphenylethylene 40. Purification by silica gel column chromatography (2\% EtOAc:hexanes) provided $56.8 \mathrm{mg}(0.17 \mathrm{mmol}, 83 \%$ yield $)$ of the title compound as a white solid. $R_{f}=0.51$ (10\% EtOAc:hexanes). IR (film): 3059 (w), 3026 (w), 2920 (w), 1728 (s), 1676 (m), 1593 (m), 1492 (s), 1340 (m), 1196 (s), 1095 (s), 954 (m) cm ${ }^{-1} .{ }^{1} \mathrm{H}$ NMR (500 MHz, $\left.\mathrm{CDCl}_{3}\right) \delta$ $7.42(\mathrm{t}, J=7.9 \mathrm{~Hz}, 2 \mathrm{H}), 7.39-7.31(\mathrm{~m}, 8 \mathrm{H}), 7.29-7.20(\mathrm{~m}, 3 \mathrm{H}), 7.16(\mathrm{dd}, J=7.4,1.6 \mathrm{~Hz}$, $2 \mathrm{H}), 6.00(\mathrm{p}, J=2.3 \mathrm{~Hz}, 1 \mathrm{H}), 4.03(\mathrm{~d}, J=2.6 \mathrm{~Hz}, 2 \mathrm{H}), 3.72(\mathrm{~d}, J=2.2 \mathrm{~Hz}, 2 \mathrm{H}) .{ }^{13} \mathrm{C}$ NMR $\left(125 \mathrm{MHz}, \mathrm{CDCl}_{3}\right) \delta 164.6,164.0,150.8,148.2,129.5,128.6,126.5,126.3,125.7,121.9$, 112.9, 48.1, 48.0, 46.7. Mp 100-101 ${ }^{\circ} \mathrm{C}$. HRMS (EI-MS): calcd for $\mathrm{C}_{24} \mathrm{H}_{20} \mathrm{O}_{2}$ $[\mathrm{M}]^{+}$340.1458; found 340.1459.

Phenyl 2-(3-(4-Methoxyphenyl)-3-phenylcyclobutylidene)acetate [30]-This compound was prepared according to General Procedure B utilizing phenyl 2,3-butadienoate 1 and 1-methoxy-4-(1-phenylvinyl)-benzene 42. Purification by silica gel column chromatography (2-5\% EtOAc:hexanes, gradient) provided $52.2 \mathrm{mg}(0.17 \mathrm{mmol}, 84 \%$ yield) of the title compound as a viscous yellow oil. $R_{f}=0.22$ (10\% EtOAc:hexanes). IR (film): 3060 (m), 2959 (w), 2835 (w), 1728 (s), 1675 (s), 1511 (s), 1493 (m), 1341 (m), 1248 (s), $1196(\mathrm{~s}), 1095(\mathrm{~s}) \mathrm{cm}^{-1} .{ }^{1} \mathrm{H}$ NMR $\left(400 \mathrm{MHz}, \mathrm{CDCl}_{3}\right) \delta$ 7.43-7.36 (m, 2H), 7.35-7.27 (m, 4H), 7.25-7.17 (m, 4H), 7.15-7.10 (m, 2H), 6.89-6.83 (m, 2H), $5.97(\mathrm{t}, J=2.3 \mathrm{~Hz}, 1 \mathrm{H})$, $3.96(\mathrm{~d}, J=2.6 \mathrm{~Hz}, 2 \mathrm{H}), 3.79(\mathrm{~s}, 3 \mathrm{H}), 3.73-3.57(\mathrm{~m}, 2 \mathrm{H}) .{ }^{13} \mathrm{C} \mathrm{NMR}\left(100 \mathrm{MHz}, \mathrm{CDCl}_{3}\right) \delta$ 164.6, 164.3, 158.0, 150.8, 148.5, 140.3, 129.5, 128.6, 127.6, 126.4, 126.2, 125.7, 121.9, 114.0, 112.9, 55.4, 48.1, 47.4, 46.8. HRMS (EI-MS): calcd for $\mathrm{C}_{25} \mathrm{H}_{22} \mathrm{O}_{3}[\mathrm{M}]^{+} 370.1563$; found 370.1579 .

Phenyl 2-(Spiro[3.5]nonan-2-ylidene)acetate [31]-This compound was prepared according to General Procedure A utilizing phenyl 2,3-butadienoate 1 and methylenecyclohexane 36. Purification by silica gel column chromatography ( $2 \%$ EtOAc:hexanes) provided $40.9 \mathrm{mg}(0.16 \mathrm{mmol}, 80 \%$ yield $)$ of the title compound as a clear colorless oil. $R_{f}=0.52$ (10\% EtOAc:hexanes). IR (film): $2922(\mathrm{~m}), 2852(\mathrm{~m}), 1730$ (s), 1672 (m), $1595(\mathrm{~m}), 1492(\mathrm{~m}), 1336(\mathrm{~m}), 1196(\mathrm{~s}), 1151(\mathrm{~s}), 1085(\mathrm{~m}) \mathrm{cm}^{-1} .{ }^{1} \mathrm{H}$ NMR (500 MHz, $\left.\mathrm{CDCl}_{3}\right) \delta 7.37(\mathrm{t}, J=7.9 \mathrm{~Hz}, 2 \mathrm{H}), 7.21(\mathrm{t}, J=7.5 \mathrm{~Hz}, 1 \mathrm{H}), 7.13-7.09(\mathrm{~m}, 2 \mathrm{H}), 5.89(\mathrm{p}, J=$ $2.3 \mathrm{~Hz}, 1 \mathrm{H}), 2.90-2.85(\mathrm{~m}, 2 \mathrm{H}), 2.61-2.54(\mathrm{~m}, 2 \mathrm{H}), 1.58-1.51(\mathrm{~m}, 4 \mathrm{H}), 1.48-1.35(\mathrm{~m}$, $6 \mathrm{H}) .{ }^{13} \mathrm{C} \mathrm{NMR}\left(100 \mathrm{MHz}, \mathrm{CDCl}_{3}\right) \delta 167.0,164.8,151.1,129.4,125.5,121.9,113.6,45.1$, 43.8, 37.9, 37.1, 25.9, 23.4.HRMS (CI-MS): calcd for $\mathrm{C}_{17} \mathrm{H}_{21} \mathrm{O}_{2}[\mathrm{M}+\mathrm{H}]^{+}$257.1536; found 257.1531 . 
Phenyl 2-(6-Phenylspiro[3.3] heptan-2-ylidene)acetate [32]-This compound was prepared according to General Procedure A utilizing phenyl 2,3-butadienoate 1 and (3methylenecyclobutyl)benzene. Purification by silica gel column chromatography ( $2 \%$ EtOAc:hexanes) provided $30.0 \mathrm{mg}(0.08 \mathrm{mmol}, 40 \%$ yield, $1.3: 1 \mathrm{dr})$ of the title compound as a clear colorless oil. $R_{f}=0.45$ (10\% EtOAc:hexanes). IR (film): 3061 (w), 3027 (w), 2953 (m), 2923 (m), 2852 (m), 1729 (s), 1673 (m), 1594 (m), 1493 (s), 1338 (m), 1199 (s), 1079 (m) $\mathrm{cm}^{-1}$. Because of overlapping signals of the inseparable diastereomers, integrations for all peaks may not be accurate. Notation of diastereomers is given where possible. ${ }^{1} \mathrm{H}$ NMR (400 MHz, $\mathrm{CDCl}_{3}$, mixture of diastereomers) $\delta 7.39(\mathrm{td}, J=7.7,4.7 \mathrm{~Hz}, 4 \mathrm{H}), 7.35-7.27(\mathrm{~m}$, $4 \mathrm{H}), 7.26-7.17(\mathrm{~m}, 8 \mathrm{H}), 7.16-7.08(\mathrm{~m}, 4 \mathrm{H}), 5.90(\mathrm{t}, J=2.3 \mathrm{~Hz}, 1 \mathrm{H}$, minor diastereomer), $5.87(\mathrm{t}, J=2.3 \mathrm{~Hz}, 1 \mathrm{H}$, major diastereomer), $3.53-3.42(\mathrm{~m}, 2 \mathrm{H}$, major diastereomer), 3.38 (d, $J=2.7 \mathrm{~Hz}, 2 \mathrm{H}$, minor diastereomer), 3.17 (d, $J=2.6 \mathrm{~Hz}, 2 \mathrm{H}$, minor diastereomer), 3.09 (d, $J=2.1 \mathrm{~Hz}, 2 \mathrm{H}$, major diastereomer), 2.88 (d, $J=2.4 \mathrm{~Hz}, 2 \mathrm{H}$ ), 2.53 (dddd, $J=12.1,9.3$, 5.8, $2.5 \mathrm{~Hz}, 4 \mathrm{H}), 2.28$ (dddd, $J=12.3,9.4,5.8,2.5 \mathrm{~Hz}, 4 \mathrm{H}) .{ }^{13} \mathrm{C} \mathrm{NMR}\left(100 \mathrm{MHz}, \mathrm{CDCl}_{3}\right.$, mixture of diastereomers) $\delta 166.0,166.0,164.8,150.8,145.3,145.2,129.5,129.5,128.4$, $128.4,126.5,126.1,126.0,125.7,125.7,121.9,121.9,112.7,47.1,45.8,45.6,44.5,41.8$, 36.2, 36.1, 34.5, 34.4. HRMS (CI-MS): calcd for $\mathrm{C}_{21} \mathrm{H}_{21} \mathrm{O}_{2}[\mathrm{M}+\mathrm{H}]^{+}$305.1536; found 305.1532 .

\section{Further Functionalization of Products}

2-(syn-3-Phenylcyclobutyl)ethan-1-ol [33]-This compound was synthesized according to either of two separate hydrogenation procedures given below.

Procedure for Reduction Using in Situ-Generated NHCCu-H Complex- $\mathrm{IPrCuCl}$ ( $9.80 \mathrm{mg}, 0.02 \mathrm{mmol}, 0.10$ equiv) $)^{28}$ and $\mathrm{NaO}^{t} \mathrm{Bu}(2.00 \mathrm{mg}, 0.02 \mathrm{mmol}, 0.10$ equiv) were weighed out in a $\mathrm{N}_{2}$-filled glovebox to a flame-dried screw cap vial. The vial was capped with a septum and removed from the glovebox. Toluene $(1.00 \mathrm{~mL}, 0.10 \mathrm{M})$ was added, and the solution was stirred for $5 \mathrm{~min}$ before the addition of PMHS $(48.0 \mu \mathrm{L}, 0.80 \mathrm{mmol}, 4.00$ equiv). The resulting black solution was stirred for $10 \mathrm{~min}$ at room temperature. A solution of $14\left(52.0 \mathrm{mg}, 0.20 \mathrm{mmol}, 1.00\right.$ equiv) and ${ }^{t} \mathrm{BuOH}(77.0 \mu \mathrm{L}, 0.80 \mathrm{mmol}, 4.00$ equiv) in $\mathrm{PhMe}(1 \mathrm{~mL}, 0.50 \mathrm{M})$ was added to the reaction flask; the septum was quickly replaced with a screw cap and allowed to stir at room temperature for $3 \mathrm{~h}$. The reaction was quenched with $\mathrm{H}_{2} \mathrm{O}(2 \mathrm{~mL})$ and extracted with EtOAc $(3 \times 1 \mathrm{~mL})$. The combined organic extracts were washed with brine, dried over $\mathrm{MgSO}_{4}$, filtered, and concentrated. The crude ester was dissolved in THF $(1 \mathrm{~mL}, 0.20 \mathrm{M})$ and cooled to $-45^{\circ} \mathrm{C}$. Lithium aluminum hydride $(1.00 \mathrm{M}$ in THF, $600 \mu \mathrm{L}, 0.60 \mathrm{mmol}, 3.00$ equiv) was added slowly, and upon complete addition, the cooling bath was replaced with an ice water bath. The reaction was allowed to stir for $2 \mathrm{~h}$ at $0{ }^{\circ} \mathrm{C}$ before carefully quenching with Rochelle's salt (sat.). The biphasic mixture was allowed to stir until two distinct layers formed ( $10 \mathrm{~min})$, and the layers separated. The aqueous phase was extracted with $\mathrm{Et}_{2} \mathrm{O}(4 \times 1 \mathrm{~mL})$. The combined organic extracts were washed with brine, dried over $\mathrm{MgSO}_{4}$, filtered, and concentrated. Purification by silica gel column chromatography (5-20\% EtOAc:hexanes, gradient) provided $25.7 \mathrm{mg}(0.15 \mathrm{mmol}$, $73 \%$ yield, $2: 1 \mathrm{dr}$ ) of the title compound as a clear colorless liquid. Diastereomeric ratio is reported as the ratio of the crude reaction products. $R_{f}=0.10$ (20\% EtOAc:hexanes). IR (film): 3400 (br, s), 3059 (m), 3027 (w), 2958 (m), 2929 (m), 1650 (s), 1494 (m), 1431 (m), 
$1265(\mathrm{~s}), 1046(\mathrm{~m}) \mathrm{cm}^{-1}$. Because of overlapping signals of the diastereomers, integrations for all peaks may not be accurate. Notation of diastereomers is given where possible. ${ }^{1} \mathrm{HNMR}\left(600 \mathrm{MHz}, \mathrm{CDCl}_{3}\right.$, major diasteromer) $\delta 7.33-7.27(\mathrm{~m}, 3 \mathrm{H}$, major/minor overlap), 7.27-7.24 (m, 1H, major/minor overlap), 7.21-7.16 (m, 3H, major/minor overlap), 3.66-3.62 (m, 3H, major/minor overlap), 3.45-3.33 (m, 1H), 2.57-2.50 (m, 2H), 2.47-2.29 $(\mathrm{m}, 2 \mathrm{H}), 1.83-1.74(\mathrm{~m}, 2 \mathrm{H}), 1.71(\mathrm{q}, J=6.9 \mathrm{~Hz}, 2 \mathrm{H}) .{ }^{1} \mathrm{H}$ NMR $\left(600 \mathrm{MHz}, \mathrm{CDCl}_{3}\right.$, minor diasteromer) $\delta$ 7.33-7.27 (m, 3H, major/minor overlap), 7.27-7.24 (m, 1H, major/minor overlap), 7.21-7.16 (m, 3H, major/minor overlap), $3.68(\mathrm{t}, J=6.7 \mathrm{~Hz}, 1 \mathrm{H}), 3.66-3.62(\mathrm{~m}$, $3 \mathrm{H}$, major/minor overlap), 2.47-2.29 (m, 2H, major/minor overlap), 2.18-2.12 (m, 2H), 1.89 (q, $J=7.0 \mathrm{~Hz}, 2 \mathrm{H}) .{ }^{13} \mathrm{C}$ NMR $\left(100 \mathrm{MHz}, \mathrm{CDCl}_{3}\right.$, mixture of isomers) $\delta 146.0,128.4,128.3$, 126.5, 126.5, 125.9, 125.8, 61.7, 61.5, 40.1, 39.2, 36.9, 36.7, 36.2, 34.2, 28.8, 28.0. HRMS (CI-MS): calcd for $\mathrm{C}_{12} \mathrm{H}_{16} \mathrm{O}_{1}[\mathrm{M}]^{+}$176.1196; found 176.1204.

Procedure for Heterogeneous Hydrogenation- $\alpha, \beta$-Unsaturated ester 14 (132 mg, $0.50 \mathrm{mmol}, 1.00$ equiv) was added to a $15 \mathrm{~mL}$ round-bottom flask under an atmosphere of air. EtOH (absolute, $5.00 \mathrm{~mL}, 0.10 \mathrm{M}$ ) was added followed by activated $\mathrm{Pd} / \mathrm{C}(6.5 \mathrm{mg})$, and the atmosphere was purged with $\mathrm{H}_{2}$. The resulting black solution was allowed to stir at ambient temperature under an atmosphere of $\mathrm{H}_{2}$ for $3 \mathrm{~h}$. Upon complete reaction, the reaction mixture was filtered through Celite into a $25 \mathrm{~mL}$ round-bottom flask, and the solvent was removed under reduced pressure. The atmosphere of the flask was replaced with $\mathrm{N}_{2}$, and the clear colorless residue was dissolved in THF (5.00 mL, $\left.0.10 \mathrm{M}\right)$. The resulting solution was cooled to $-45^{\circ} \mathrm{C}$. Lithium aluminum hydride $(1.00 \mathrm{M}$ in THF, $1.50 \mathrm{~mL}, 1.50$ mmol, 3.00 equiv) was added slowly, and upon complete addition, the cooling bath was replaced with an ice water bath. The reaction was allowed to stir for $2 \mathrm{~h}$ at $0{ }^{\circ} \mathrm{C}$ before carefully quenching with Rochelle's salt (sat.). The biphasic mixture was allowed to stir until two distinct layers formed ( 10 min), and the layers separated. The aqueous phase was extracted with $\mathrm{Et}_{2} \mathrm{O}(4 \times 1 \mathrm{~mL})$. The combined organic extracts were washed with brine, dried over $\mathrm{MgSO}_{4}$, filtered, and concentrated. The colorless crude residue was purified by silica gel column chromatography (5-20\% EtOAc:hexanes, gradient) to provide $65.3 \mathrm{mg}$ ( $0.37 \mathrm{mmol}, 74 \%$ yield, $2.2: 1 \mathrm{dr}$ ) of the title compound as a clear colorless liquid.

Diastereomeric ratio is reported as the ratio of the crude reaction products. All spectral data was consistent with that of 33 generated by the reduction procedure outlined above.

Phenyl 2-(syn-1,3-Diphenylcyclobutyl)acetate [34]-[Rh(COD)-Cl $]_{2}(5.00 \mathrm{mg}, 0.01$ mmol, 0.05 equiv) was weighed out in a $\mathrm{N}_{2}$-filled glovebox to a flame-dried screw cap vial and dissolved in dioxane $(900 \mu \mathrm{L}, 0.01 \mathrm{M})$. The vial was capped with a septum and removed from the glovebox. $\mathrm{KOH}\left(1.5 \mathrm{M}\right.$ in $\mathrm{H}_{2} \mathrm{O}, 175 \mu \mathrm{L}, 2.60 \mathrm{mmol}, 1.30$ equiv) was added followed by phenyl 2-(3-phenylcyclobutylidene)acetate $(53.0 \mathrm{mg}, 0.20 \mathrm{mmol}, 1.00$ equiv) in dioxane $(320 \mu \mathrm{L}, 0.63 \mathrm{M}) . \mathrm{PhB}(\mathrm{OH})_{2}(49.0 \mathrm{mg}, 0.40 \mathrm{mmol}, 2.00$ equiv) was added in one portion; the septum was replaced with a screw cap, and the reaction mixture was stirred for $16 \mathrm{~h}$ at room temperature. The reaction was quenched with brine $(2 \mathrm{~mL})$ and extracted with EtOAc $(3 \times 1 \mathrm{~mL})$. The combined organic extracts were washed with brine, dried over $\mathrm{MgSO}_{4}$, filtered, and concentrated. Purification by silica gel column chromatography (2\% EtOAc:hexanes) provided $43.2 \mathrm{mg}(0.15 \mathrm{mmol}$, $73 \%$ yield, $3.2: 1 \mathrm{dr})$ of the title compound as a white solid. Diastereomeric ratio is reported as the ratio of the crude reaction products. 
$R_{f}=0.50$ (10\% EtOAc:hexanes). IR (film): 3061 (w), 3025 (w), 2990 (w), 2933 (w), 1751 (s), 1593 (w), 1493 (m), 1275 (s), 1261 (s), $1196(\mathrm{~m}), 1146(\mathrm{~m}) \mathrm{cm}^{-1}$. Because of overlapping signals of the diastereomers, integrations for all peaks may not be accurate. Notation of diastereomers is given where possible. ${ }^{1} \mathrm{H}$ NMR $\left(600 \mathrm{MHz}, \mathrm{CDCl}_{3}\right.$, major diastereomer) $\delta$ 7.37-7.32 (m, 2H, major/minor overlap), 7.32-7.23 (m, 8H, major/minor overlap), 7.23-7.20 (m, 2H, major/minor overlap), 7.19-7.14 (m, 2H, major/minor overlap), 6.80-6.76 (m, 2H, major/minor overlap), 3.82-3.74 (m, 1H), 3.17 (s, 2H), 3.05-2.99 (m, 2H), 2.67-2.59 (m, 4H, major/minor overlap). ${ }^{1} \mathrm{H}$ NMR (600 MHz, $\mathrm{CDCl}_{3}$, minor diastereomer) $\delta 7.59-7.54(\mathrm{~m}, 1 \mathrm{H}), 7.43(\mathrm{t}, J=7.7 \mathrm{~Hz}, 1 \mathrm{H}), 7.37-7.32(\mathrm{~m}, 2 \mathrm{H}$, major/minor overlap), 7.32-7.23 (m, 8H, major/minor overlap), 7.23-7.20 (m, 2H, major/minor overlap), 7.19-7.14 (m, 2H, major/minor overlap), 6.80-6.76 (m, 2H, major/minor overlap), 3.43 (p, $J$ $=9.2 \mathrm{~Hz}, 1 \mathrm{H}), 3.11-3.05(\mathrm{~m}, 1 \mathrm{H}), 2.98(\mathrm{~s}, 2 \mathrm{H}), 2.67-2.59(\mathrm{~m}, 4 \mathrm{H}$, major/minor overlap). ${ }^{13} \mathrm{C} \mathrm{NMR}\left(100 \mathrm{MHz}, \mathrm{CDCl}_{3}\right.$, mixture of diastereomers) $\delta 170.1,150.5,148.6$, 145.0, 129.4, 128.6, 128.5, 128.4, 128.42 127.0, $126.6\left({ }^{13} \mathrm{C}\right), 126.3,126.2,126.1,125.8$, 125.8, 121.7, 49.6, 46.2, 41.6, 41.4, 41.3, 40.3, 34.7, 34.0. Mp 105-108 ${ }^{\circ} \mathrm{C}$. HRMS (CIMS): calcd for $\mathrm{C}_{24} \mathrm{H}_{23} \mathrm{O}_{2}[\mathrm{M}+\mathrm{H}]^{+} 343.1693$; found 343.1702 .

\section{Phenyl 2-(syn-3-Phenyl-1-(phenylthio)cyclobutyl)acetate [35]-IMesCuCl (8.10} $\mathrm{mg}, 0.02 \mathrm{mmol}, 0.10$ equiv $)^{29}$ and $\mathrm{NaSPh}(2.60 \mathrm{mg}, 0.02 \mathrm{mmol}, 0.10$ equiv) were weighed out in a $\mathrm{N}_{2}$-filled glovebox to a flame-dried screw cap vial. The vial was capped with a septum and removed from the glovebox. PhMe $(1.00 \mathrm{~mL}, 0.02 \mathrm{M})$ was added, and the solution was stirred for $5 \mathrm{~min}$ at room temperature. A solution of $14(52.0 \mathrm{mg}, 0.20 \mathrm{mmol}$, 1.00 equiv) and $\mathrm{PhSH}(31 \mu \mathrm{L}, 0.30 \mathrm{mmol}, 1.50$ equiv) in $\mathrm{PhMe}(500 \mu \mathrm{L}, 0.10 \mathrm{M})$ was added to the reaction vial. The septum was replaced with a screw cap, and the reaction was allowed to stir for $24 \mathrm{~h}$. The reaction was quenched with $\mathrm{H}_{2} \mathrm{O}(2 \mathrm{~mL})$ and extracted with EtOAc $(3 \times$ $1 \mathrm{~mL}$ ). The combined organic extracts were washed with brine, dried over $\mathrm{MgSO}_{4}$, filtered, and concentrated. Purification by silica gel chromatography (2\% EtOAc:hexanes) provided $43.6 \mathrm{mg}$ (58\% yield, 2:1 dr) of the title compound as a clear colorless liquid. Diastereomeric ratio is reported as the ratio of the crude reaction products. $R_{f}=0.50$ (10\% EtOAc:hexanes). Further purification by silica gel chromatography (2-5\% $\mathrm{Et}_{2} \mathrm{O}$ :pentane, gradient) to provide analytically pure quantities of each diastereomer was performed for characterization purposes. $R_{\text {fmajor }}=0.35, R_{\text {fminor }}=0.41\left(10 \% \mathrm{Et}_{2} \mathrm{O}\right.$ :pentane). IR (film): $3058(\mathrm{w}), 3025(\mathrm{w})$, 2957 (w), 2937 (w), 1753 (s), 1593 (m), 1493 (s), 1455 (m), 1438 (m), 1338 (w), 1275 (s), 1261 (s), 1193 (s), 1160 (s), 1029 (w) cm ${ }^{-1}$. ${ }^{1} \mathrm{H}$ NMR (600 MHz, $\mathrm{CDCl}_{3}$, major diastereomer) $\delta 7.60-7.56(\mathrm{~m}, 2 \mathrm{H}), 7.43-7.34(\mathrm{~m}, 5 \mathrm{H}), 7.31-7.26(\mathrm{~m}, 3 \mathrm{H}), 7.21-7.17(\mathrm{~m}$, $3 \mathrm{H}), 7.15$ (d, $J=7.6 \mathrm{~Hz}, 2 \mathrm{H}), 3.56$ (p, $J=9.1 \mathrm{~Hz}, 1 \mathrm{H}), 3.09$ (s, 2H), 2.90-2.84 (m, 2H), 2.67-2.59 (m, 2H). ${ }^{1} \mathrm{H}$ NMR (600 MHz, $\mathrm{CDCl}_{3}$, minor diastereomer) $\delta 7.68(\mathrm{dd}, J=6.6$, $3.0 \mathrm{~Hz}, 2 \mathrm{H}), 7.41-7.37(\mathrm{~m}, 5 \mathrm{H}), 7.31(\mathrm{t}, J=7.5 \mathrm{~Hz}, 2 \mathrm{H}), 7.25-7.22(\mathrm{~m}, 3 \mathrm{H}), 7.20(\mathrm{t}, J=7.3$ $\mathrm{Hz}, 1 \mathrm{H}), 7.16-7.12(\mathrm{~m}, 2 \mathrm{H}), 4.02(\mathrm{p}, J=9.0 \mathrm{~Hz}, 1 \mathrm{H}), 2.91(\mathrm{~s}, 2 \mathrm{H}), 2.75-2.68(\mathrm{~m}, 2 \mathrm{H}), 2.52-$ $2.46(\mathrm{~m}, 2 \mathrm{H}) .{ }^{13} \mathrm{C} \mathrm{NMR}\left(125 \mathrm{MHz}, \mathrm{CDCl}_{3}\right.$, major diastereomer) $\delta 169.6,150.8,144.4$, 135.9, 132.3, 129.6, 129.2, 128.8, 128.6, 126.8, 126.4, 126.1, 121.9, 46.6, 43.2, 42.3, 34.1. ${ }^{13} \mathrm{C}$ NMR (125 MHz, $\mathrm{CDCl}_{3}$, minor diastereomer) $\delta 169.3,150.8,144.5,136.4,132.6$, 129.6, 129.2, 129.0, 128.6, 126.6, 126.3, 126.1, 121.9, 48.1, 46.3, 41.1, 34.8. HRMS (EIMS): calcd for $\mathrm{C}_{24} \mathrm{H}_{22} \mathrm{O}_{2} \mathrm{~S}_{1}[\mathrm{M}]^{+}$374.1335; found 374.1327 . 


\section{Synthesis of Alkenes}

General Procedure C. ${ }^{30}-\mathrm{Ph}_{3} \mathrm{PCH}_{3} \mathrm{Br}$ (1.20 equiv) was added to a flame-dried roundbottom flask, evacuated, backfilled with $\mathrm{N}_{2}$ three times, and suspended in $\mathrm{Et}_{2} \mathrm{O}(0.30 \mathrm{M})$. To this vigorously stirring heterogeneous solution was added $\mathrm{KO}^{t} \mathrm{Bu}$ (1.20 equiv), and the reaction was allowed to stirred at room temperature for $15 \mathrm{~min}$ until a bright yellow heterogeneous mixture was achieved. The resulting solution was cooled to $0{ }^{\circ} \mathrm{C}$, and the appropriate aldehyde/ketone (1.00 equiv) was added slowly so as to avoid flash boiling of the ethereal solvent. Upon complete addition, the cooling bath was removed, and the reaction was allowed to stir for $\sim 15 \mathrm{~h}$ before filtering through Celite and concentrating. The crude material was purified by silica gel column chromatography, and NMR spectra was compared to known literatures values.

Methylenecyclohexane [36] - This compound was synthesized according to General Procedure $\mathrm{C}$ and purified by silica gel column chromatography (pentane) to yield $705 \mathrm{mg}$ (7.34 mmol, 72\% yield) of the title compound as a clear colorless liquid. Spectral data were consistent with literature-reported values. ${ }^{31}{ }^{1} \mathrm{H}$ NMR $\left(400 \mathrm{MHz}, \mathrm{CDCl}_{3}\right) \delta 4.58(\mathrm{~s}, 2 \mathrm{H})$, $2.13(\mathrm{t}, J=5.3 \mathrm{~Hz}, 4 \mathrm{H}), 1.62-1.43(\mathrm{~m}, 6 \mathrm{H}) .{ }^{13} \mathrm{C} \mathrm{NMR}\left(100 \mathrm{MHz}, \mathrm{CDCl}_{3}\right) \delta 150.6,107.1$, $35.5,28.2,26.6$.

2-Bromostyrene [37]-This compound was synthesized according to General Procedure $\mathrm{C}$ and purified by silica gel column chromatography (hexanes) to yield $920 \mathrm{mg}$ (5.03 mmol, 93\% yield) of the title compound as a clear colorless liquid. Spectral data were consistent with literature-reported values. ${ }^{32}{ }^{1} \mathrm{H}$ NMR $\left(400 \mathrm{MHz}, \mathrm{CDCl}_{3}\right) \delta$ 7.58-7.52 (m, 2H), 7.31$7.26(\mathrm{~m}, 1 \mathrm{H}), 7.16-7.01(\mathrm{~m}, 2 \mathrm{H}), 5.71(\mathrm{dd}, J=17.5,1.1 \mathrm{~Hz}, 1 \mathrm{H}), 5.37$ (dd, $J=11.0,1.1 \mathrm{~Hz}$, 1H). ${ }^{13} \mathrm{C} \mathrm{NMR}\left(100 \mathrm{MHz}, \mathrm{CDCl}_{3}\right) \delta 137.6,135.9,133.0,129.2,127.6,126.9,123.7,116.8$.

3-Bromostyrene [38]-This compound was synthesized according to General Procedure $\mathrm{C}$ and purified by silica gel column chromatography (hexanes) to yield $890 \mathrm{mg}(4.86 \mathrm{mmol}$, $90 \%$ yield) of the title compound as a clear colorless liquid. Spectral data were consistent with literature-reported values. ${ }^{33}{ }^{1} \mathrm{H} \mathrm{NMR}\left(400 \mathrm{MHz}, \mathrm{CDCl}_{3}\right) \delta 7.56(\mathrm{~s}, 1 \mathrm{H}), 7.41-7.35(\mathrm{~m}$, 1H), 7.35-7.29 (m, 1H), 7.19 (t, $J=7.8 \mathrm{~Hz}, 1 \mathrm{H}), 6.65$ (dd, $J=17.6,10.9 \mathrm{~Hz}, 1 \mathrm{H}), 5.76(\mathrm{~d}, J$ $=17.6 \mathrm{~Hz}, 1 \mathrm{H}), 5.30(\mathrm{~d}, J=10.9 \mathrm{~Hz}, 1 \mathrm{H}) .{ }^{13} \mathrm{C} \mathrm{NMR}\left(100 \mathrm{MHz}, \mathrm{CDCl}_{3}\right) \delta 139.8,135.6$, $130.8,130.2,129.3,125.0,122.9,115.5$.

4-Bromostyrene [39]—This compound was synthesized according to General Procedure $\mathrm{C}$ and purified by silica gel column chromatography (hexanes) to yield $940 \mathrm{mg}(5.13 \mathrm{mmol}$, $95 \%$ yield) of the title compound as a clear colorless liquid. Spectral data were consistent with literature-reported values. ${ }^{34}{ }^{1} \mathrm{H}$ NMR $\left(400 \mathrm{MHz}, \mathrm{CDCl}_{3}\right) \delta 7.48-7.38(\mathrm{~m}, 2 \mathrm{H}), 7.31-$ $7.22(\mathrm{~m}, 2 \mathrm{H}), 6.64(\mathrm{dd}, J=17.6,10.9 \mathrm{~Hz}, 1 \mathrm{H}), 5.73(\mathrm{~d}, J=17.6 \mathrm{~Hz}, 1 \mathrm{H}), 5.26$ (dd, $J=11.0$, $1.5 \mathrm{~Hz}, 1 \mathrm{H}) .{ }^{13} \mathrm{C}$ NMR $\left(100 \mathrm{MHz}, \mathrm{CDCl}_{3}\right) \delta 136.6,135.9,131.8,128.0,121.7,114.7$.

Ethene-1,1-diyldibenzene [40]-This compound was synthesized according to General Procedure $\mathrm{C}$ and purified by silica gel column chromatography (hexanes) to yield $861 \mathrm{mg}$ ( $4.77 \mathrm{mmol}, 87 \%$ yield) of the title compound as a clear colorless liquid. Spectral data were consistent with literature-reported values. ${ }^{35}{ }^{1} \mathrm{H}$ NMR $\left(400 \mathrm{MHz}, \mathrm{CDCl}_{3}\right) \delta$ 7.54-7.28 (m, 
$10 \mathrm{H}), 5.49(\mathrm{~d}, J=5.7 \mathrm{~Hz}, 2 \mathrm{H}) .{ }^{13} \mathrm{C}$ NMR $\left(100 \mathrm{MHz}, \mathrm{CDCl}_{3}\right) \delta 150.2,141.6,128.4,128.3$, 127.8, 114.4.

4-Vinylphenyl 4-Methylbenzenesulfonate [41]—This compound was synthesized according to General Procedure $\mathrm{C}$ and purified by silica gel column chromatography (5$20 \%$ EtOAc:hexanes, gradient) to yield $695 \mathrm{mg}$ ( $2.53 \mathrm{mmol}, 70 \%$ yield $)$ of the title compound as a white solid. Spectral data were consistent with literature-reported values. ${ }^{36}{ }^{1} \mathrm{H}$ NMR $\left(400 \mathrm{MHz}, \mathrm{CDCl}_{3}\right)$ \& 7.72-7.63 (m, 2H), 7.31-7.27 (m, 4H), 6.97-6.89 $(\mathrm{m}, 2 \mathrm{H}), 6.70-6.59(\mathrm{~m}, 1 \mathrm{H}), 5.68(\mathrm{~d}, J=17.6 \mathrm{~Hz}, 1 \mathrm{H}), 5.26(\mathrm{dd}, J=10.8,1.7 \mathrm{~Hz}, 1 \mathrm{H}), 2.44$ $(\mathrm{d}, J=2.1 \mathrm{~Hz}, 3 \mathrm{H}) .{ }^{13} \mathrm{C} \mathrm{NMR}\left(100 \mathrm{MHz}, \mathrm{CDCl}_{3}\right) \delta 149.1,145.4,136.6,135.5,132.4$, $129.8,128.5,127.3,122.5,114.9,21.7$.

1-Methoxy-4-(1-phenylvinyl)benzene [42]-This compound was synthesized according to General Procedure $\mathrm{C}$ and purified by silica gel column chromatography (210\% EtOAc:hexanes, gradient) to yield $812 \mathrm{mg}$ ( $3.86 \mathrm{mmol}, 82 \%$ yield) of the title compound as a white solid ( $82 \%$ yield). Spectral data were consistent with literaturereported values. ${ }^{37}{ }^{1} \mathrm{H}$ NMR (400 MHz, $\left.\mathrm{CDCl}_{3}\right) \delta 7.35-7.27(\mathrm{~m}, 5 \mathrm{H}), 7.27-7.21(\mathrm{~m}, 2 \mathrm{H})$, 6.87-6.79 (m, 2H), 5.35 (dd, $J=17.2,1.3 \mathrm{~Hz}, 2 \mathrm{H}), 3.80$ (s, 3H). ${ }^{13} \mathrm{C} \mathrm{NMR}(100 \mathrm{MHz}$, $\left.\mathrm{CDCl}_{3}\right) \delta 159.4,149.6,141.9,134.0,129.5,128.4,128.2,127.8,113.6,113.0,55.4$.

General Procedure D. ${ }^{38}$ - The corresponding boronic acid (1.00 equiv) and pinacol (1.00 equiv) were added to a flame-dried flask and purged with $\mathrm{N}_{2}$. THF $(0.20 \mathrm{M})$ was added to dissolve the solids, and two spatula tips of $\mathrm{MgSO}_{4}$ were added to the mixture. The reaction was allowed to stir for $2 \mathrm{~h}$ at room temperature, filtered through Celite, and concentrated to give the desired product, which could be used without further purification.

3-Vinylphenylboronic Acid Pinacol Ester [43]—This compound was synthesized according to General Procedure D from (3-vinylphenyl)-boronic acid to provide $1.53 \mathrm{~g}$ (6.65 mmol, $98 \%$ yield) of the title compound as a clear colorless oil. Spectral data were consistent with literature-reported values. ${ }^{38}{ }^{1} \mathrm{H} \mathrm{NMR}\left(500 \mathrm{MHz}, \mathrm{CDCl}_{3}\right) \delta 7.84(\mathrm{~s}, 1 \mathrm{H})$, $7.70(\mathrm{~d}, J=7.3 \mathrm{~Hz}, 1 \mathrm{H}), 7.52(\mathrm{dt}, J=7.7,1.6 \mathrm{~Hz}, 1 \mathrm{H}), 7.34(\mathrm{t}, J=7.5 \mathrm{~Hz}, 1 \mathrm{H}), 6.73(\mathrm{dd}, J=$ 17.6, $10.9 \mathrm{~Hz}, 1 \mathrm{H}), 5.79(\mathrm{dd}, J=17.6,1.0 \mathrm{~Hz}, 1 \mathrm{H}), 5.24(\mathrm{~d}, J=10.9 \mathrm{~Hz}, 1 \mathrm{H}), 1.35$ (s, $12 \mathrm{H}) .{ }^{13} \mathrm{C} \mathrm{NMR}\left(100.0 \mathrm{MHz}, \mathrm{CDCl}_{3}\right) \delta 136.9,136.8,134.2,132.7,128.9,127.9,113.9$, 83.9, 24.9.

4-Vinylphenylboronic Acid Pinacol Ester [44]—This compound was synthesized according to General Procedure D from (4-vinylphenyl)-boronic acid to provide $1.54 \mathrm{~g}$ (6.69 $\mathrm{mmol}, 99 \%$ yield) as a clear colorless liquid. Spectral data were consistent with literaturereported values. ${ }^{38}{ }^{1} \mathrm{H}$ NMR $\left(600 \mathrm{MHz}, \mathrm{CDCl}_{3}\right) \delta 7.77(\mathrm{~d}, J=7.7 \mathrm{~Hz}, 2 \mathrm{H}), 7.41(\mathrm{~d}, J=7.8$ $\mathrm{Hz}, 2 \mathrm{H}), 6.73$ (dd, $J=17.6,10.8 \mathrm{~Hz}, 1 \mathrm{H}), 5.81$ (d, $J=17.6 \mathrm{~Hz}, 1 \mathrm{H}), 5.29$ (d, $J=10.9 \mathrm{~Hz}$, $1 \mathrm{H}), 1.35(\mathrm{~s}, 12 \mathrm{H}) .{ }^{13} \mathrm{C} \mathrm{NMR}\left(100.0 \mathrm{MHz}, \mathrm{CDCl}_{3}\right) \delta 140.2,136.9,135.0,125.5,114.9$, 83.8, 24.9.

1-Phenylvinylboronic Acid Pinacol Ester [45]—This compound was synthesized according to General Procedure D from (1-phenylvinyl)-boronic acid to provide $1.53 \mathrm{~g}$ (6.65 $\mathrm{mmol}, 98 \%$ yield) of the title compound as an orange oil. Spectral data were consistent with 
literature-reported values. ${ }^{39}{ }^{1} \mathrm{H} \mathrm{NMR}\left(500 \mathrm{MHz}, \mathrm{CDCl}_{3}\right) \delta 7.48(\mathrm{~d}, J=7.3,2 \mathrm{H}), 7.31(\mathrm{t}, J=$ 7.5, 2H), $7.25(\mathrm{~m}, 1 \mathrm{H}), 6.06(\mathrm{~m}, 2 \mathrm{H}), 1.32(\mathrm{~s}, 12 \mathrm{H}) .{ }^{13} \mathrm{C} \mathrm{NMR}\left(125 \mathrm{MHz}, \mathrm{CDCl}_{3}\right) \delta 131.0$, 128.3, 127.3, 127.2, 83.9, 25.0, 24.7.

\section{Supplementary Material}

Refer to Web version on PubMed Central for supplementary material.

\section{Acknowledgments}

We thank Indiana University and the National Institutes of Health (1R01GM110131) for generous financial support. M.K.B. is the recipient of a 2016 Amgen Young Investigator Award.

\section{References}

1. Belluš D, Ernst B. Angew. Chem., Int. Ed. Engl. 1988; 27:797.

2. Namyslo JC, Kaufmann DE. Chem. Rev. 2003; 103:1485. [PubMed: 12683789]

3. Lee-Ruff E, Mladenova G. Chem. Rev. 2003; 103:1449. [PubMed: 12683788]

4. (a) Izquierdo S, Rua F, Sbai A, Parella T, Álvarez-Larena Á, Branchadell V, Ortuño RM. J. Org. Chem. 2005; 70:7963. [PubMed: 16277316] (b) Berlicki L, Kaske M, Gutiérrez-Abad R, Bernhardt G, Illa O, Ortuño RM, Cabrele C, Buschauer A, Reiser O. J. Med. Chem. 2013; 56:8422. [PubMed: 24090364]

5. Xu Y, Conner ML, Brown MK. Angew. Chem., Int. Ed. 2015; 54:11918.

6. Brimioulle R, Lenhart D, Maturi MM, Bach T. Angew. Chem., Int. Ed. 2015; 54:3872.

7. Wager TT, Pettersen BA, Schmidt AW, Spracklin DK, Mente S, Butler TW, Howard H, Lettiere DJ, Rubitski DM, Wong DF, Nedza FM, Nelson FR, Rollema H, Raggon JW, Aubrecht J, Freeman JK, Marcek JM, Cianfrogna J, Cook KW, James LC, Chatman LA, Iredale PA, Banker MJ, Homiski ML, Munzner JB, Chandrasekaran RY. J. Med. Chem. 2011; 54:7602. [PubMed: 21928839]

8. Chalmers BA, Xing H, Houston S, Clark C, Ghassabian S, Kuo A, Cao B, Reitsma A, Murray C-EP, Stok JE, Boyle GM, Pierce CJ, Littler SW, Winkler DA, Bernhardt PV, Pasay C, De Voss JJ, McCarthy J, Parsons PG, Walter GH, Smith MT, Cooper HM, Nilsson SK, Tsanaktsidis J, Savage GP, Williams CM. Angew. Chem. 2016; 128:3644.

9. Stepan AF, Subramanyam C, Efremov IV, Dutra JK, O’Sullivan TJ, DiRico KJ, McDonald WS, Won A, Dorff PH, Nolan CE, Becker SL, Pustilnik LR, Riddell DR, Kauffman GW, Kormos BL, Zhang L, Lu Y, Capetta SH, Green ME, Karki K, Sibley E, Atchison KP, Hallgren AJ, Oborski CE, Robshaw AE, Sneed B, O’Donnell CJ. J. Med. Chem. 2012; 55:3414. [PubMed: 22420884]

10. Radchenko DS, Grygorenko OO, Komarov IV. Tetrahedron: Asymmetry. 2008; 19:2924.

11. Staudinger H. Ber. Dtsch. Chem. Ges. 1905; 38:1735.

12. Krepski LR, Hassner A. J. Org. Chem. 1978; 43:2879.

13. Danheiser, RL. Science of Synthesis: Compounds with Four and Three Carbon Heteroatom Bonds. Vol. 23. Georg Thieme Verlag KG; Germany: 2006.

14. Lawlor MD, Lee TW, Danheiser RL. J. Org. Chem. 2000; 65:4375. [PubMed: 10891141]

15. Hoyt JM, Schmidt VA, Tondreau AM, Chirik PJ. Science. 2015; 349:960. [PubMed: 26315433]

16. Conner ML, Xu Y, Brown MK. J. Am. Chem. Soc. 2015; 137:3482. [PubMed: 25756948]

17. For other [2 + 2] cycloadditions between alkene and electrondeficient allenes, see: Padwa A, Lipka H, Watterson SH, Murphree SS. J. Org. Chem. 2003; 68:6238. [PubMed: 12895056] Zhao J-F, Loh T-P. Angew. Chem., Int. Ed. 2009; 48:7232.

18. (a) Snider BB, Spindell DK. J. Org. Chem. 1980; 45:5017.(b) Hoffmann HMR, Ismail ZM, Weber A. Tetrahedron Lett. 1981; 22:1953.(c) Snider BB, Ron E. J. Org. Chem. 1986; 51:3643.

19. For thermal [2+2] cycloaddition between enol ethers and allenoates, see: Jung ME, Nishimura N, Novack AR. J. Am. Chem. Soc. 2005; 127:11206. [PubMed: 16089425] Jung ME, Murakami M. Org. Lett. 2006; 8:5857. [PubMed: 17134290] 
20. Cowen BJ, Saunders LB, Miller SJ. J. Am. Chem. Soc. 2009; 131:6105. [PubMed: 19354290]

21. For comparison, the reaction of methyl 2,3-butadienoate with 1-hexene, styrene, and allylBpin proceeds in 10,60, and $20 \%$ yield, respectively. This further highlights the increased reactivity of phenyl 2,3-butadienoate relative to that of methyl 2,3-butadienoate (this allene was used in the Snider and Hoffman studies ${ }^{18}$ ).

22. (a) Mlynarski SN, Karns AS, Morken JP. J. Am. Chem. Soc. 2012; 134:16449. [PubMed: 23002712] (b) Balieu S, Hallett GE, Burns M, Bootwicha T, Studley J, Aggarwal VK. J. Am. Chem. Soc. 2015; 137:4398. [PubMed: 25625684]

23. Jurkauskas V, Sadighi JP, Buchwald SL. Org. Lett. 2003; 5:2417. [PubMed: 12841744]

24. Wuitschik G, Rogers-Evans M, Mueller K, Fischer H, Wagner B, Schuler F, Polonchuk L, Carreira EM. Angew. Chem., Int. Ed. 2006; 45:7736. For a review of rhodium-catalyzed conjugate addition in synthesis, see: Edwards HJ, Hargrave JD, Penrose SD, Frost CG. Chem. Soc. Rev. 2010; 39:2093. [PubMed: 20407730]

25. Delp SA, Munro-Leighton C, Goj LA, Ramírez MA, Gunnoe TB, Petersen JL, Boyle PD. Inorg. Chem. 2007; 46:2365. [PubMed: 17338519]

26. Hong BC, Lin CW, Liao WK, Lee GH. Org. Lett. 2013; 15:6258. [PubMed: 24266326]

27. Abell AD, Hoult DA, Morris KM, Taylor JM, Trent JO. J. Org. Chem. 1993; 58:1531.

28. Prepared according to: Kaur H, Zinn FK, Stevens ED, Nolan S. Organometallics. 2004; 23:1157.

29. Prepared according to: Xie W, Chang S. Angew. Chem., Int. Ed. 2016; 55:1876.

30. Adapted procedure from: Hui BW, Chiba S. Org. Lett. 2009; 11:729. [PubMed: 19123787]

31. Brecknell DJ, Carman RM, Edwards RA, Hansford KA, Karoli T, Robinson WT. Aust. J. Chem. 1997; 50:589.

32. Grigg RD, Van Hoveln R, Schomaker JMJ. Am. Chem. Soc. 2012; 134:16131.

33. Yao C, Li Q, Wang M, Ning X, Kang Y. Chem. Commun. 2015; 51:7729.

34. Huang J, Lin Z, Chen D. Org. Lett. 2012; 14:22. [PubMed: 22149492]

35. Oh CH, Jung HH, Kim KS, Kim N. Angew. Chem., Int. Ed. 2003; 42:805.

36. Su YH, Wu Z, Tian SK. Chem. Commun. 2013; 49:6528.

37. Al-huniti MH, Lepore SD. Org. Lett. 2014; 16:4154. [PubMed: 25061668]

38. Adapted procedure from: Baltus CB, Chuckowree IS, Press NJ, Day IJ, Coles SJ, Tizzard GJ, Spencer J. Tetrahedron Lett. 2013; 54:1211.

39. Guan W, Michael AK, McIntosh ML, Selfridge LK, Scott JP, Clark TB. J. Org. Chem. 2014; 79:7199. [PubMed: 24915498] 

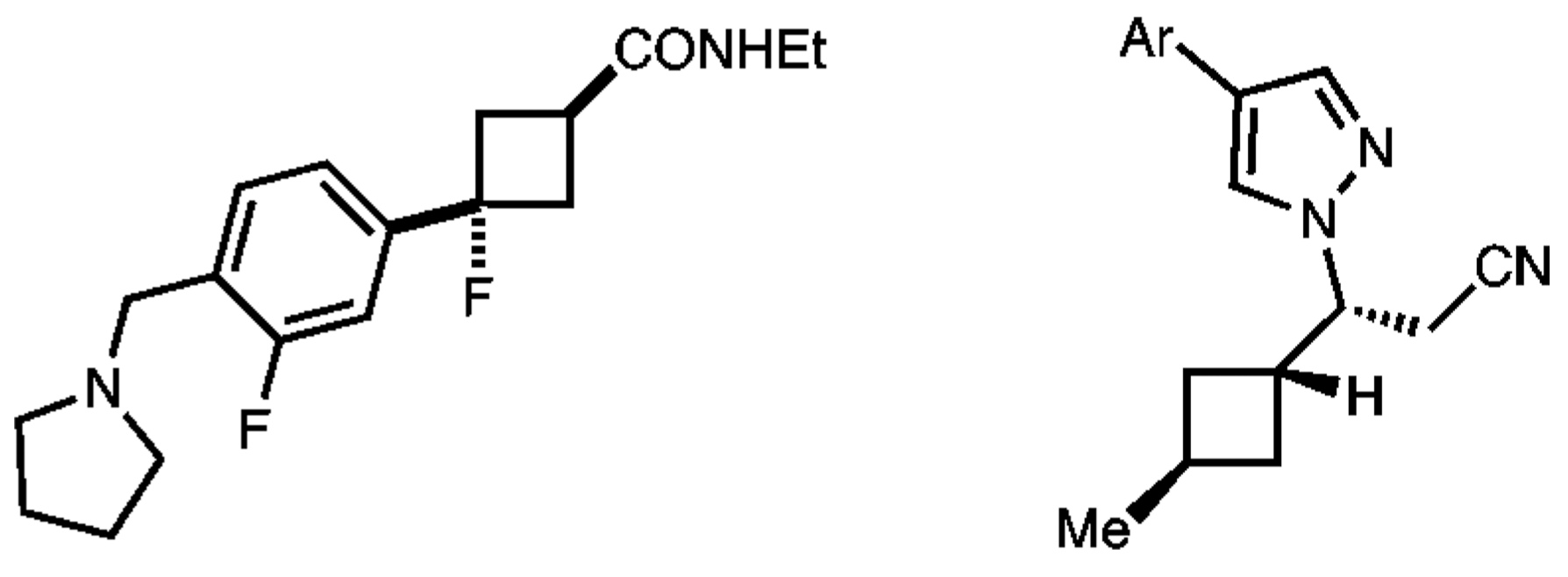

Figure 1.

Representative 1,3-substituted cyclobutanes. 
A) Snider (1980)<smiles>[R7]C(=C)[OH2+]</smiles>

B) Hoffman (1981)<smiles>C=CC[AsH3]</smiles><smiles>C=C=CC(=O)OC</smiles>

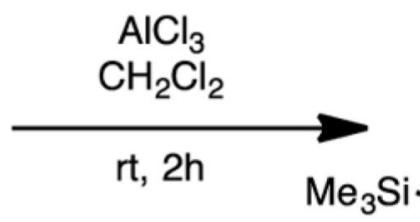<smiles>CCC1CC(=CC(=O)OC)C1</smiles>

C) Brown (2015)

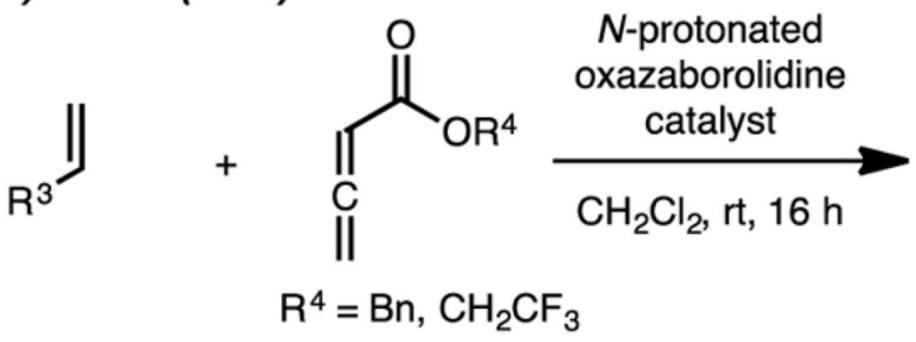

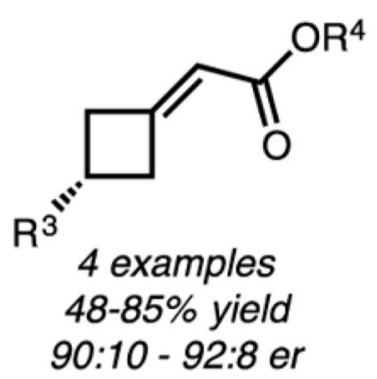

D) This Work<smiles>[R]C(=C)[CH+]=C=CC(=O)Oc1ccccc1</smiles><smiles>[R]C1([R])CC(=CC(=O)Oc2ccccc2)C1</smiles>

\section{- Broad Scope

Scheme 1.

$[2+2]$ Cycloaddition of Allenoates and Alkenes 
A) Wittig reaction route to allenoates<smiles>O=C(/C=[PH+]\c1ccccc1)c1ccccc1</smiles><smiles>CC[C@@H](N(CC)CC)C(Cl)(Cl)Cl</smiles><smiles>C=C=CC(=O)Oc1ccccc1</smiles><smiles></smiles>

Liquid at room temperature -Stable at $-20^{\circ} \mathrm{C}$ for months

B) Use of 2-naphthyl derived allenoate<smiles>C=C=CC(=O)OCCCCCCC(=O)OCC</smiles>
(2 equiv)
Ar = 2-napthyl

C) Use of $\alpha$-bromoallenoate<smiles>CCCCC(=O)OC(=O)C=C1CC(CCCC)C1</smiles>

$72 \%$ yield Ar = 2-napthyl<smiles>C=C=C(Br)C(=O)Oc1ccccc1</smiles>

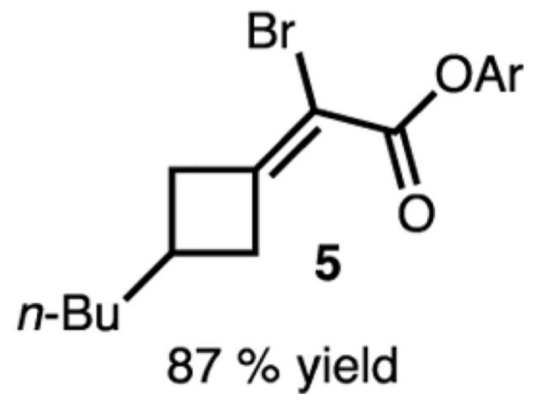

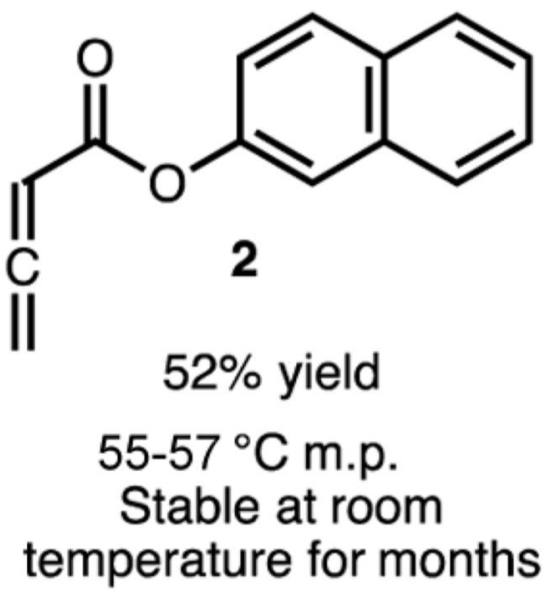

Scheme 2.

Synthesis and Use of Allenoates 


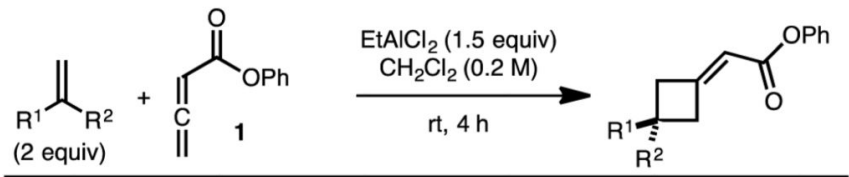

- Monosubstituted Alkenes
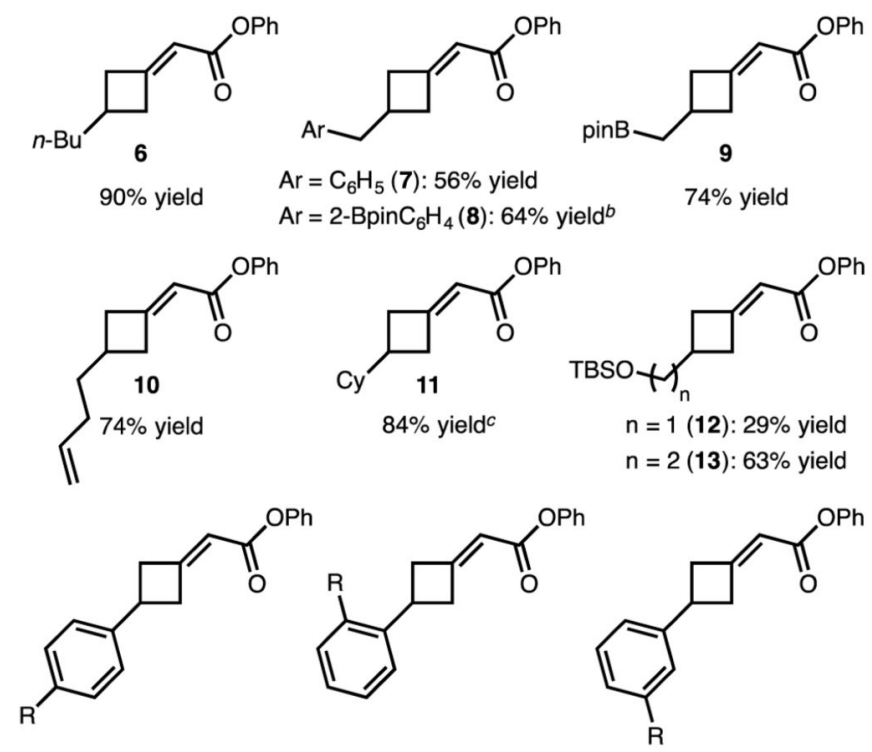

$\mathrm{R}=\mathrm{H}(\mathbf{1 4 )}): 90 \%$ yield $^{d}$

$R=F(15): 61 \%$ yield

$\mathrm{R}=\mathrm{Br}(\mathbf{1 6 )}: \mathbf{6 9 \%}$ yield

$R=\operatorname{Me~(20):~} 62 \%$ yield $\quad R=B r(23): 78 \%$ yield

$R=$ Bpin (17): $38 \%$ yield

$R=B r(21): 93 \%$ yield $\quad R=B p i n(24): 70 \%$ yield

$\mathrm{R}=$ Bpin (22): $37 \%$ yield

$R=$ OAc (18): $66 \%$ yield

$R=$ OTs (19): $69 \%$ yield

1,1-Disubstituted Alkenes
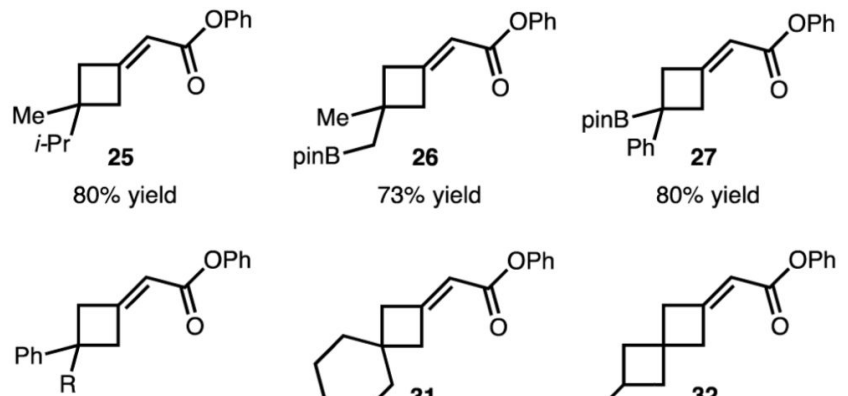

$R=M e(28): 98 \%$ yield

$\mathrm{R}=\mathrm{Ph}(29): 83 \%$ yield

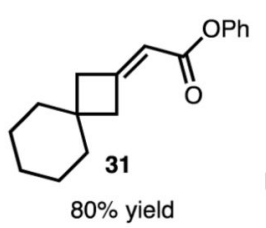

$\mathrm{R}=4-\mathrm{OMeC}_{A} \mathrm{H}_{A}(30): 84 \%$ vield

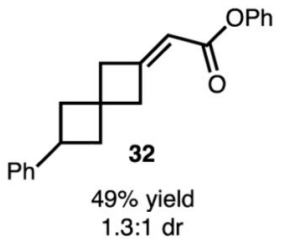

Scheme 3.

$[2+2]$ Cycloadditions with Terminal Alkenes ${ }^{a}$

${ }^{a}$ Yield represents the average of at least two separate experiments $(0.2 \mathrm{mmol}$ scale). ${ }^{b}$ Reaction run for $16 \mathrm{~h}(2.1 \mathrm{mmol}) .{ }^{c}$ Formed as a $13: 1$ mixture of regioisomers. ${ }^{d}$ Reaction run with $1.0 \mathrm{~g}(6.5 \mathrm{mmol})$ of 1 .

J Org Chem. Author manuscript; available in PMC 2017 September 02. 
1) $10 \mathrm{~mol} \% \mathrm{IPrCuCl}$

$\mathrm{NaOt}$-Bu

$t$-BuOH, PHMS

toluene, $\mathrm{rt}, 3 \mathrm{~h}$
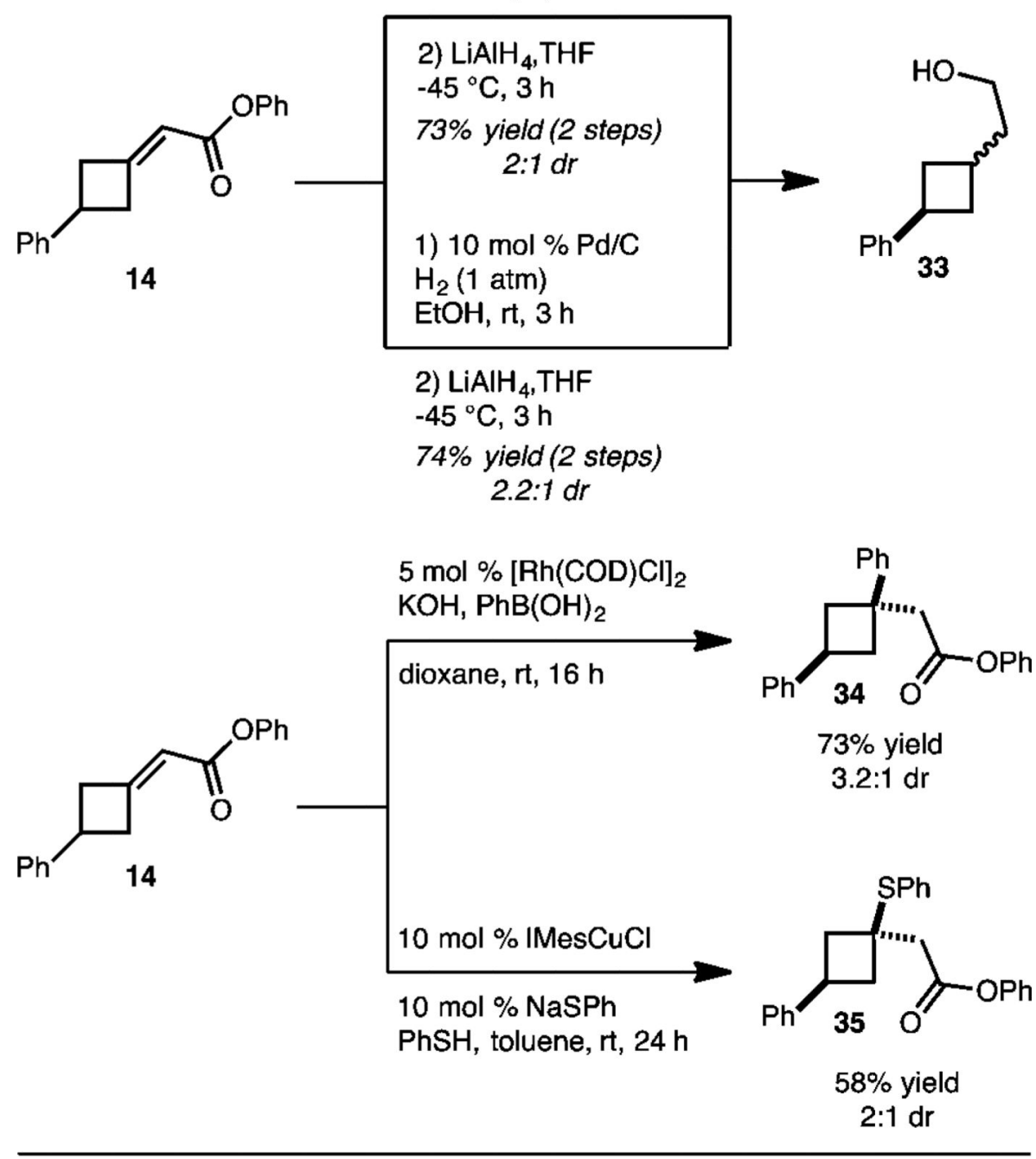

- Putative Basis for Selectivity

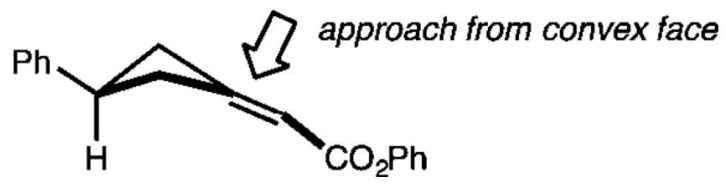

Scheme 4.

Further Functionalization of Products 


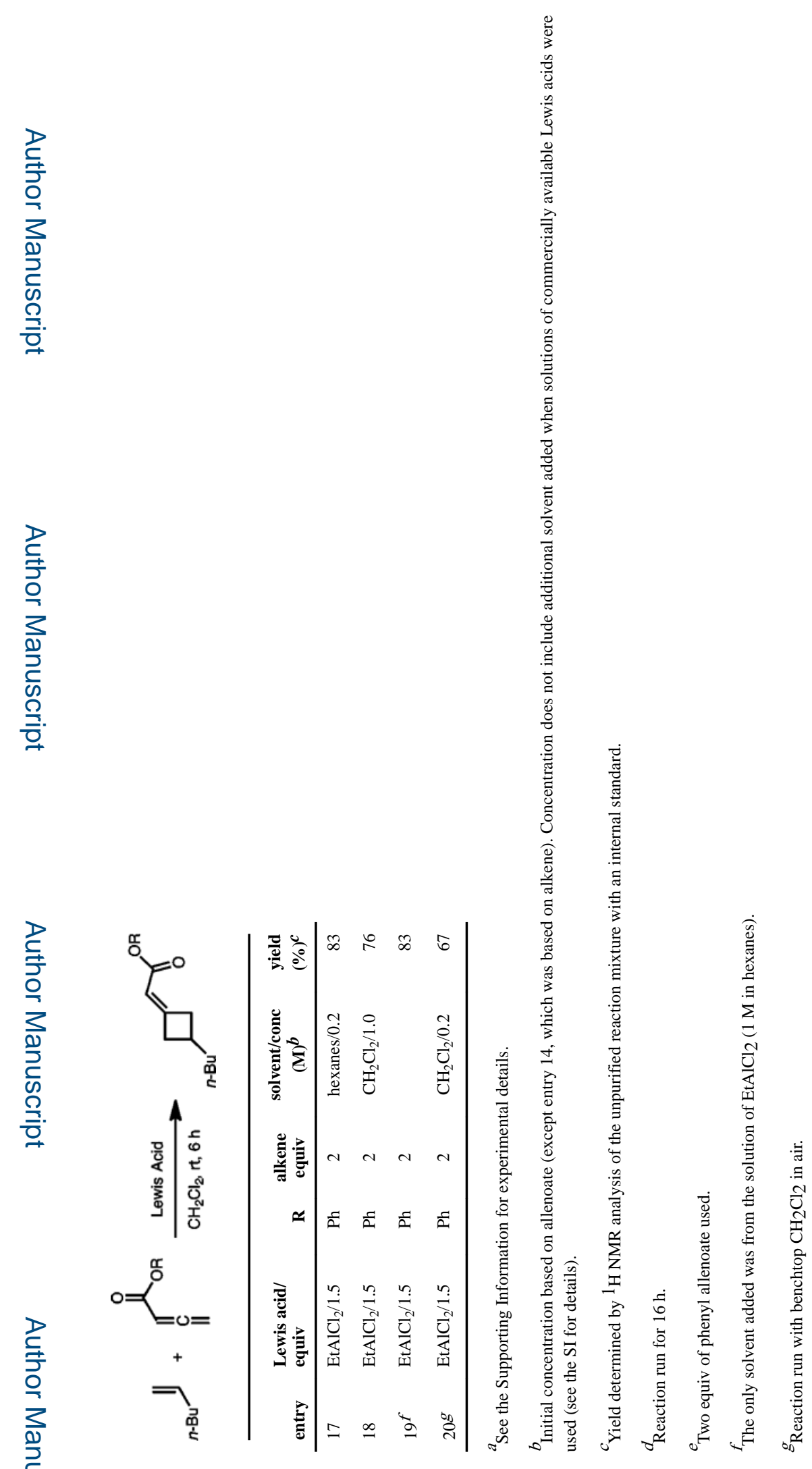

